\title{
INSTABILITY OF A BINARY LIQUID FILM FLOWING DOWN A SLIPPERY INCLINED HEATED PLATE
}

\author{
by \\ Eglal Ellaban \\ Master of Applied Mathematics, Suez Canal University, 2003 \\ Bachelor of Science, Suez Canal University, 1993
}

\author{
A thesis \\ presented to Ryerson University \\ in partial fulfillment of the \\ requirements for the degree of \\ Master of Mathematics \\ in the Program of \\ Applied Mathematics
}

Toronto, Ontario, Canada, 2016

(C)Eglal Ellaban 2016 


\section{Author's Declaration}

I hereby declare that I am the sole author of this thesis. This is a true copy of the thesis, including any required final revisions, as accepted by my examiners.

I authorize Ryerson University to lend this thesis to other institutions or individuals for the purpose of scholarly research.

I further authorize Ryerson University to reproduce this thesis by photocopying or by other means, in total or in part, at the request of other institutions or individuals for the purpose of scholarly research.

I understand that my thesis may be made electronically available to the public. 
Instability of a binary liquid film flowing down a slippery inclined heated plate

Master of Mathematics 2016

Eglal Ellaban

Applied Mathematics

Ryerson University

In this thesis we studied the stability of a binary liquid film flowing down a heated porous inclined plate. It is assumed that the heating induces concentration differences in the liquid mixture (Soret effect), which together with the differences in temperature affects the surface tension. A mathematical model is constructed by coupling the Navier-Stokes equations governing the flow with equations for the concentration and temperature. The effect of substrate permeability is incorporated by applying a specific slip condition at the bottom of the liquid layer. We carry out a linear stability analysis in order to obtain the critical conditions for the onset of instability. We used a Chebyshev spectral collocation method to obtain numerical solutions to the resulting Orr-Sommerfeld type equations. We also obtained an asymptotic solution which yielded an expression for the state of neutral stability of long perturbations as a function of the parameters controlling the problem. We present our findings by illustrating and interpreting our results for the critical Reynolds number for instability. 


\section{Acknowledgements}

First and foremost I would like to gratefully acknowledge my supervisor Dr. Jean-Paul Pascal for his guidance, encouragement and valuable support he has provided throughout my thesis. I owe a debt of gratitude to him for his valuable time and knowledge.

My special gratitude goes to my thesis committee members: Dr. Silvana Ilie, Dr. Katrin Rohlf and Dr. Garnet Ord for their encouragement and valuable suggestions. In a special way I would like to thank Ryerson University for offering me the special chance to study in the Applied Mathematics program.

Last but not the least, I would like to thank my parents who taught me patience and hard work. Thanks also go to my brother Khaled for his encouragement and sacrifice throughout my academic

studies. I would also like to thank my kids: Alaa, Moemen, Youssef and Abd ElRahman for their love and support. 


\section{Dedication}

I would like to dedicate this thesis to my parents and my brother Khaled. 


\section{Contents}

1 Introduction 1

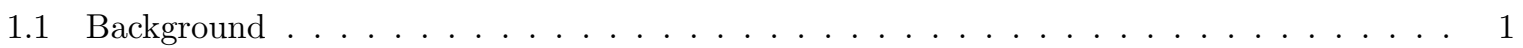

1.2 Description of the problem . . . . . . . . . . . . . . . . . . . . . 3

2 Governing Equations $\mathbf{5}$

2.1 Dimensional equations . . . . . . . . . . . . . . . . . . . . . 5

2.2 Non-Dimensional equations ............................. 9

3 Linear Stability Analysis 13

3.1 Equilibrium solution . . . . . . . . . . . . . . . . . . . . . . . . . . 13

3.2 Linear stability theory . . . . . . . . . . . . . . . . . . . . . . . . . . 14

3.3 Asymptotic solution . . . . . . . . . . . . . . . . . . . . . . . . . . . 19

3.4 Numerical solution ..... . . . . . . . . . . . . . . . . . . . . . . . 24

4 Conclusions $\quad 38$

\begin{tabular}{ll} 
References & 40 \\
\hline
\end{tabular} 


\section{List of Figures}

1.1 Schematic representation of a thin film flowing down a porous inclined heated plane. . . . 3

3.1 Neutral stability curves for different values of $M a$ with $\cot \theta=1, \operatorname{Pr}=7, B i=1, K a=$

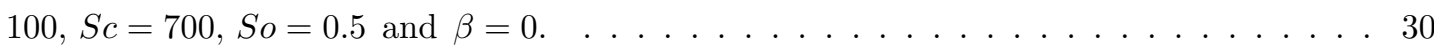

3.2 Neutral stability curves for different values of $M a$ with $\cot \theta=1, \operatorname{Pr}=7, B i=1, K a=$

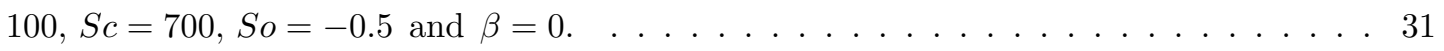

3.3 Neutral stability curves for different values of $M a$ with $\cot \theta=1, \operatorname{Pr}=7, B i=1, K a=$

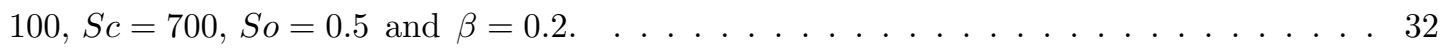

3.4 Neutral stability curves for different values of $M a$ with $\cot \theta=1, \operatorname{Pr}=7, B i=1, K a=$

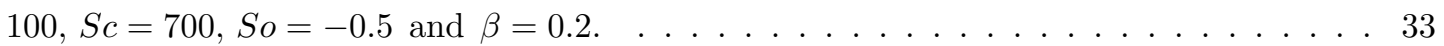

$3.5 R e_{\text {crit }}$ as function of $M a$ with $\cot \theta=1, \operatorname{Pr}=7, B i=1, K a=100, S c=700$ and $S o=$

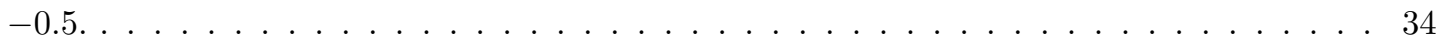

3.6 Re $e_{\text {crit }}$ as function of $M a$ with $\cot \theta=1, \operatorname{Pr}=7, K a=100, S c=700, S o=-0.5$ and $\beta=$

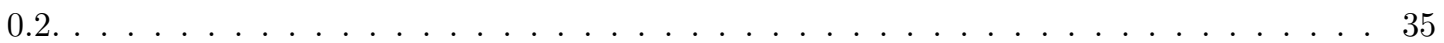

3.7 Re crit as function of $M a$ with $\cot \theta=1, P r=7, B i=1, K a=100, S c=700$ and $\beta=0.2 .36$

$3.8 R e_{c r i t}$ as function of $M a$ with $\cot \theta=1, \operatorname{Pr}=7, B i=1, S c=700, S o=-0.5$ and $\beta=0.2 .37$ 


\section{Chapter 1}

\section{Introduction}

\section{$1.1 \quad$ Background}

Liquid films flowing down an inclined plane have widespread applications in both environmental phenomena and industrial settings. The formation of interfacial waves due to hydrodynamic instability exhibits complicated dynamics in spite of the simplicity of the flow configuration. Therefore, this type of flow has received significant interest and has fascinated many researchers.

The first experiment was conducted by Kapitza [1] in 1948 who investigated the development of interfacial waves in the flow of thin films down an inclined plate. In 1949, an additional study was performed experimentally and theoretically by Kapitza and Kapitza [2]. They succeeded in establishing the basic fundamental systematic study in this type of problem. Furthermore, they identified a dimensionless flow parameter known as the Kapitza number which is considered a significant parameter in the study of the formation of interfacial waves. This Kapitza number is defined by a combination of surface tension, kinematic viscosity and gravitational force. Later, more studies were conducted by Benjamin [3] and Yih [4] who performed a linear stability analysis to investigate the long-wave instability of isothermal falling films. The critical Reynolds number was calculated using a perturbation solution to the Orr-Sommerfeld equation. Since then, many researchers have pursued various extensions to the original problem established by the Kapitza father and son team.

The impact of heating on the flow down an inclined plate has recently been investigated. In 2003, Kalliadasis et al. [5] assumed that surface tension depends linearly on temperature and examined the instability of the flow over a uniformly heated inclined plate using a first order integral-boundary-layer approximation. This yields a reduced-dimensionality model that is amenable to linear and nonlinear analyses, however it does not provide good accuracy in predicting the onset of instability when compared to the linear analysis of the full equations and experimental observations. The work presented by Ruyer-Quil et al. [6] and Scheid et al. [7] used a second-order weighted residual approach to obtain 
a more accurate reduced model that exactly reproduces the critical Reynolds number for the onset of instability. Trevelyan et al. [8] applied the weighted residual model assuming both constant temperature and constant heat flux as a bottom boundary condition. They found that in the long-wave limit, heating destabilizes the flow in both cases. The study presented by D'Alessio et al. [9] showed that the thermocapillary effect destabilizes the flow over a heated wavy inclined surface.

The first study on the linear stability of a thin Newtonian flow down a porous incline plate was presented by Pascal [10]. He constructed a theoretical model by using a "one-sided" model which is an incorporation of the flow equations and a boundary condition describing the permeability of the substrate. He used the slip condition formulated by Beavers and Joseph [11] to describe the boundary condition at the porous substrate and discarded the filtration flow in the porous medium assuming that it is much slower than the clear flow. Later, Sadiq et al. [12] investigated the effect of surface tension on the Newtonian film flow using an extension to the one-sided model taken by Pascal. They obtained a Benney-type equation, then a weakly nonlinear analysis was carried out to determine how the permeability of the porous medium impacts the formation of the interfacial waves. The accuracy of the one-sided model was examined by Liu and Liu [13] who concluded that using this approach generates more accurate results when the permeability is low or moderate, particularly if the porous substrate is sufficiently thin.

In addition, Pascal [14] implemented the one-sided model to examine the instability of a nonNewtonian flow over a porous inclined plate. He performed a depth integrating strategy to obtain a onedimensional model, then a linear and nonlinear analysis were conducted to determine the onset of instability. In another study, Pascal and D'Alessio [15] performed the weighted residual method to investigate the instability in a gravity-driven flow over a permeable wavy surface. They found that the bottom topography coupled with strong surface tension destabilizes the flow. Sadiq et al. [16] presented a paper investigating the influence of heating and permeability on the instability of the flow. They obtained an Orr-Sommerfeld type equation and both numerical and asymptotic solutions were calculated to investigate the influences of heating and permeability on the onset of instability. Their research includes an important comparison between their results and previous results obtained by applying the weighted residual method.

Binary liquids refer to mixtures of two liquids such as ethanol-water mixtures and liquid-metal alloys. The liquid comprising the largest part of the mixture acts as the solvent, while the other is the solute. It turns out that, if heated, binary liquids are subject to the Soret effect, whereby temperature differences induce a flux of molecules of the solute leading to concentration variations. The influence of the Soret effect in the horizontal case has been presented in several studies. In 1979, Takashima [17] examined the impact of the Soret effect on the P-mode by extending Pearson's linear stability analysis [18] to binary liquid. More recently, another study was performed by Joo [19] who used a model with a deformable free surface heated from above or below and investigated the influence of the Soret effect on the proposed instability, however the hydrostatic effect was not included in his model. Podolny et al. [20] published a 
paper investigating the influence of the Soret effect on the S-mode in the presence of heat flux. The first work to examine the instability of a binary liquid film flow over an uniformly heated inclined plane was presented by $\mathrm{Hu}$ et al. [21]. In their work, the system of equations for linear stability was solved using the Chebyshev collocation method. They found that the flow become more unstable with increasing the Soret number or increasing the Marangoni number.

The present study represents an extension of the work by Hu et al. [21] to also include the permeability of the plate. The objective of this thesis is to establish a theoretical model and investigate the interaction of thermosolutal-capillary effects combined with bottom permeability on the instability of the flow. We model this problem by coupling the governing flow equations with the temperature and concentration equations and employ the Beavers-Joseph condition to describe the porosity of the plate. In chapter 2 we introduce the governing equations and the corresponding boundary conditions. In chapter 3 , a linear stability analysis is conducted resulting in a Orr-Sommerfeld type equation. A numerical method is performed to produce a solution of the eigenvalue problem. An asymptotic solution is also presented in this chapter which also includes a presentation and discussion of the results, while the conclusions of the study are summarized in chapter 4 .

\subsection{Description of the problem}

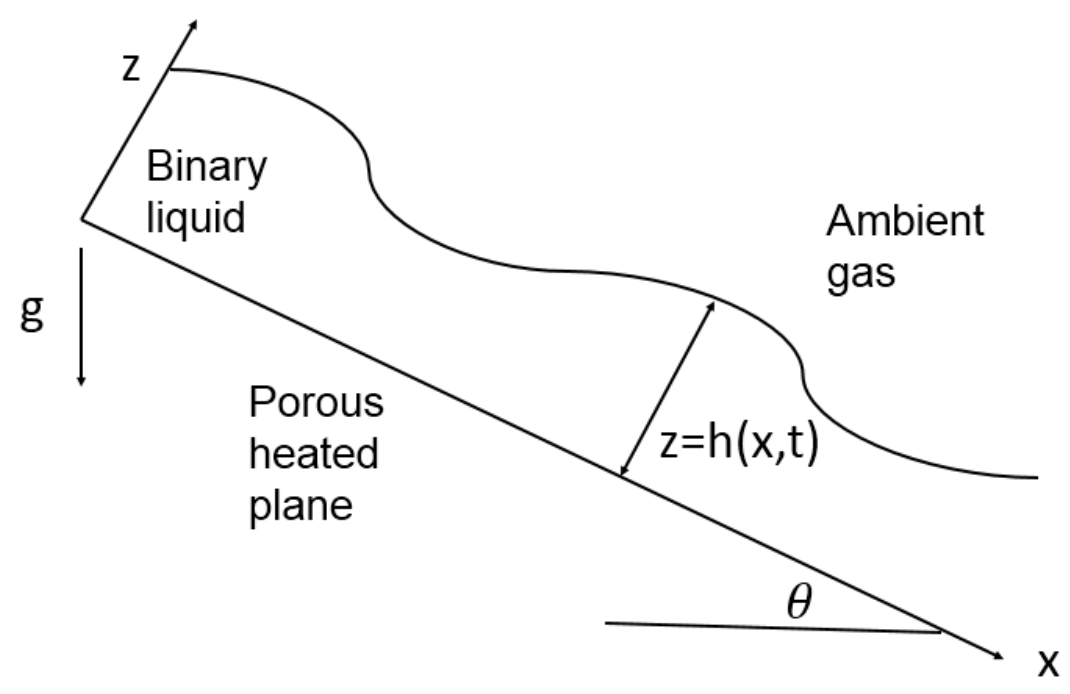

Figure 1.1: Schematic representation of a thin film flowing down a porous inclined heated plane.

We consider the gravity-driven two dimensional laminar flow of a binary liquid film over a uniformly heated porous plate inclined at an angle $\theta$ with respect to the horizontal as shown in Figure 1.1. The $x$-axis points down the incline and the $z$-axis points upwards. The velocity components in the $x$ and $z$ directions are given by $u$ and $w$ respectively while $z=h(x, t)$ refers to the thickness of the fluid. The 
temperature of the porous plate is maintained at a prescribed constant value denoted by $T_{w}$, while the ambient gas which is assumed to be motionless has a constant temperature $T_{\infty}<T_{w}$ and pressure $p_{\infty}$. 


\section{Chapter 2}

\section{Governing Equations}

\subsection{Dimensional equations}

We consider a binary liquid film flowing down a slippery inclined heated plate as illustrated in Figure 1.1. The two-dimensional equations which describe the motion of the fluid are obtained from the NavierStokes equations and continuity equation. The $x$-momentum equation can be written as

$$
\frac{\partial u}{\partial t}+u \frac{\partial u}{\partial x}+w \frac{\partial u}{\partial z}=-\frac{1}{\rho} \frac{\partial p}{\partial x}+g \sin \theta+\frac{\mu}{\rho}\left(\frac{\partial^{2} u}{\partial x^{2}}+\frac{\partial^{2} u}{\partial z^{2}}\right)
$$

while the $z$-momentum equation is given by

$$
\frac{\partial w}{\partial t}+u \frac{\partial w}{\partial x}+w \frac{\partial w}{\partial z}=-\frac{1}{\rho} \frac{\partial p}{\partial z}-g \cos \theta+\frac{\mu}{\rho}\left(\frac{\partial^{2} w}{\partial x^{2}}+\frac{\partial^{2} w}{\partial z^{2}}\right)
$$

where $p$ is the pressure, $u$ and $w$ are the streamwise velocity and cross-stream velocity respectively. The density is given by $\rho$ while $\mu$ is the dynamic viscosity. The gravitational acceleration is denoted by $g$ while $\theta$ represents the angle of inclination of the plate. The continuity equation is expressed as

$$
\frac{\partial u}{\partial x}+\frac{\partial w}{\partial z}=0
$$

According to Fourier's law the heat flux is proportional to the temperature gradient, and similarly, by Fick's law the mass flux is proportional to the concentration gradient. However, when both temperature and concentration differences exist, the heat and mass fluxes can be affected by both the temperature and concentration gradients. The dependence of the mass flux on the temperature gradient is referred to as the Soret effect, while the dependence of the heat flux on the concentration gradient is known as the Dufour effect. However, in liquid mixtures only the Soret effect is significant. Consequently the heat flux $J_{h}$ and mass flux $J_{m}$ will be expressed as

$$
J_{h}=-\lambda \nabla T
$$




$$
J_{m}=-\rho D_{m}(\nabla C+\alpha \nabla T)
$$

where $C$ is the solute concentration, $T$ is the temperature of the liquid mixture, $\lambda>0$ is the thermal conductivity, $D_{m}>0$ is the mass diffusivity, $\alpha$ is the Soret coefficient and $\nabla=\left(\frac{\partial}{\partial x}, \frac{\partial}{\partial z}\right)$. We point out that, depending on the nature of the binary liquid, the temperature-induced solute flux can be towards warmer or colder regions, and consequently the Soret coefficient can take on negative or nonnegative values.

Introducing the heat and mass fluxes into the basic advection-diffusion transport equation we obtain the following equations governing the temperature and concentration

$$
\begin{gathered}
\frac{D T}{D t}=\delta \nabla^{2} T \\
\frac{D C}{D t}=D_{m}\left(\nabla^{2} C+\alpha \nabla^{2} T\right)
\end{gathered}
$$

where $\delta$ is the thermal diffusivity and $\frac{D}{D t}=\frac{\partial}{\partial t}+u \frac{\partial}{\partial x}+w \frac{\partial}{\partial z}$.

To determine the dynamical conditions at the surface of the liquid layer, we first point out that, assuming the liquid is Newtonian, the total stress tensor can be expressed as $\vec{s}=-p \vec{I}+\vec{\tau}$, where

$$
\vec{\tau}=\mu\left[\begin{array}{cc}
2 \frac{\partial u}{\partial x} & \frac{\partial u}{\partial z}+\frac{\partial w}{\partial x} \\
\frac{\partial u}{\partial z}+\frac{\partial w}{\partial x} & 2 \frac{\partial w}{\partial z}
\end{array}\right] \text { and } \vec{I}=\left[\begin{array}{ll}
1 & 0 \\
0 & 1
\end{array}\right]
$$

The force exerted by the flow on the free surface $z=h(x, t)$, can be written as $\vec{s} \widehat{n}$ where $\widehat{n}$ is the unit normal vector which is given by

$$
\widehat{n}=\frac{1}{\sqrt{1+\left(\frac{\partial h}{\partial x}\right)^{2}}}\left[\begin{array}{c}
-\frac{\partial h}{\partial x} \\
1
\end{array}\right] .
$$

We assume the viscous stress acting on the surface due to the ambient gas to be negligible. Therefore, the force on the surface due to the flow of the liquid film is balanced by the ambient pressure and the effect of surface tension. Then the total force balance at the surface of the liquid layer can be expressed as

$$
\vec{s} \cdot \widehat{n}=-\left(p_{\infty}+2 \sigma K\right) \widehat{n}+\vec{\nabla}_{s} \sigma
$$

where $p_{\infty}$ is the ambient pressure, $\sigma$ is the surface tension, $\nabla_{s}$ is the surface gradient operator which is defined as

$$
\overrightarrow{\nabla_{s}}=\frac{1}{n^{2}}\left[\begin{array}{c}
\frac{\partial}{\partial x}+\frac{\partial h}{\partial x} \frac{\partial}{\partial z} \\
\frac{\partial h}{\partial x} \frac{\partial}{\partial x}+\left(\frac{\partial h}{\partial x}\right)^{2} \frac{\partial}{\partial z}
\end{array}\right]
$$

and $K$ is the average of the mean curvature of the free surface which is given by $K=-\frac{1}{2}\left(\vec{\nabla}_{s} \cdot \widehat{n}\right)$. The 
normal component of the equation (2.8) yields to the following equation

$$
\begin{array}{r}
p=p_{\infty}+\frac{2 \mu}{1+\left(\frac{\partial h}{\partial x}\right)^{2}}\left[\left(\frac{\partial h}{\partial x}\right)^{2} \frac{\partial u}{\partial x}-\frac{\partial h}{\partial x}\left(\frac{\partial u}{\partial z}+\frac{\partial w}{\partial x}\right)+\frac{\partial w}{\partial z}\right] \\
-\frac{\sigma \frac{\partial^{2} h}{\partial x^{2}}}{\left(1+\left(\frac{\partial h}{\partial x}\right)^{2}\right)^{\frac{3}{2}}} \quad \text { at } z=h(x, t) .
\end{array}
$$

The tangential component of the equation (2.8) can be obtained by multiplying the equation by $\widehat{t}$, the unit tangent vector, which can be written as

$$
\widehat{t}=\frac{1}{\sqrt{1+\left(\frac{\partial h}{\partial x}\right)^{2}}}\left[\begin{array}{c}
1 \\
\frac{\partial h}{\partial x}
\end{array}\right] .
$$

We thus obtain

$$
\begin{array}{r}
{\left[\frac{\partial \sigma}{\partial x}+\frac{\partial h}{\partial x}\left(\frac{\partial \sigma}{\partial z}\right)\right] \sqrt{1+\left(\frac{\partial h}{\partial x}\right)^{2}}=\mu\left[\left(1-\left(\frac{\partial h}{\partial x}\right)^{2}\right)\left(\frac{\partial u}{\partial z}+\frac{\partial w}{\partial x}\right)+2 \frac{\partial h}{\partial x}\left(\frac{\partial w}{\partial z}-\frac{\partial u}{\partial x}\right)\right]} \\
\text { at } z=h(x, t) .
\end{array}
$$

The left-hand side of this equation corresponds to the so-called Marangoni stress which is due to variation in surface tension, and pulls fluid along the surface in the direction of increasing surface tension.

The surface tension is assumed to depend linearly on both the temperature and the solute concentration and is expressed as

$$
\sigma=\sigma_{\infty}-\sigma_{t}\left(T-T_{\infty}\right)+\sigma_{c}\left(C-C_{0}\right)
$$

where $\sigma_{\infty}$ is the surface tension of the fluid at the reference values $T=T_{\infty}$ and $C=C_{0}$. The parameters $\sigma_{t}$ and $\sigma_{c}$ are defined as

$$
\sigma_{t}=-\frac{\partial \sigma}{\partial T} \quad, \quad \sigma_{c}=\frac{\partial \sigma}{\partial C}
$$

which are positive for nearly all binary liquids. So surface tension increases with concentration and decreases with temperature.

A kinematic condition for the free surface of the liquid layer can be derived from the assumption that evaporation will be neglected, i.e. the fluid particles on the surface must remain on the surface as fluid flows. The kinematic condition at the surface can be expressed as

$$
w=\frac{\partial h}{\partial t}+\frac{\partial h}{\partial x} u \quad \text { at } \quad z=h(x, t)
$$

The relation between the heat flux normal to the surface and the difference in the temperature of the 
liquid and the ambient gas can be expressed through Newton's law of cooling which is given by

$$
-\lambda \nabla T \cdot \widehat{n}=\chi\left(T-T_{\infty}\right) \quad \text { at } \quad z=h(x, t)
$$

where $\chi$ is the heat transfer coefficient and $T_{\infty}$ is the ambient temperature.

Also, using the fact that the normal mass flux of solute vanishes at the free surface, we obtain the boundary condition

$$
-\frac{\partial h}{\partial x} \frac{\partial C}{\partial x}+\frac{\partial C}{\partial z}+\alpha\left(-\frac{\partial h}{\partial x} \frac{\partial T}{\partial x}+\frac{\partial T}{\partial z}\right)=0 \quad \text { at } \quad z=h(x, t)
$$

At a fluid-porous medium interface, Beavers and Joseph [11] established experimentally that the appropriate boundary condition can be written as

$$
\begin{gathered}
\frac{\partial u}{\partial z}=\frac{\varpi}{\sqrt{\kappa}}\left(u-u_{p}\right) \quad \text { at } \quad z=0, \\
w=w_{p} \text { at } \quad z=0,
\end{gathered}
$$

where $\kappa$ is the permeability of the porous medium, $\varpi$ is a dimensionless parameter related to the structure of the porous medium, while $\left(u_{p}, w_{p}\right)$ is the Darcian mean filtration velocity in the porous medium. For sufficiently low permeability the filtration velocity can be neglected since the flow of the fluid through the porous medium is much slower than that of the clear fluid layer, then the equations (2.16) and (2.17) can be used to define boundary conditions for the velocity of the clear fluid:

$$
\begin{gathered}
\frac{\partial u}{\partial z}=\frac{\varpi}{\sqrt{\kappa}} u \quad \text { at } \quad z=0, \\
w=0 \quad \text { at } \quad z=0 .
\end{gathered}
$$

Since the normal velocity is negligible at the bottom, we also have a zero normal solute flux, which is expressed as

$$
\frac{\partial C}{\partial z}+\alpha \frac{\partial T}{\partial z}=0 \quad \text { at } \quad z=0
$$

And finally, since the substrate is maintained at temperature $T_{w}$, we have the condition

$$
T=T_{w} \quad \text { at } \quad z=0 .
$$




\subsection{Non-Dimensional equations}

The governing equations can be written in dimensionless form by using an appropriate scaling. For the length scale we choose

$$
H=\left(\frac{3 \mu Q}{\rho g \sin \theta}\right)^{\frac{1}{3}}
$$

which is the Nusselt thickness of an isothermal flow along a non-permeable surface if the flow rate is prescribed to be $Q$. As a result, we will introduce the following transformation

$$
\begin{gathered}
(x, z)=H\left(x^{*}, z^{*}\right), h=H h^{*},(u, w)=U\left(u^{*}, w^{*}\right), t=\frac{H}{U} t^{*}, \\
p-p_{\infty}=\rho U^{2} p^{*}, T=T_{\infty}+\Delta T T^{*}, C=C_{0}+\frac{\sigma_{t}}{\sigma_{c}} \Delta T C^{*}
\end{gathered}
$$

where $U=Q / H$ and $\Delta T=T_{w}-T_{\infty}$. Applying the scaling described above to the momentum equations in the $x$ and $z$ directions gives respectively

$$
\frac{\partial\left(U u^{*}\right)}{\partial\left(\frac{H}{U} t^{*}\right)}+\left(U u^{*}\right) \frac{\partial\left(U u^{*}\right)}{\partial\left(H x^{*}\right)}+\left(U w^{*}\right) \frac{\partial\left(U u^{*}\right)}{\partial\left(H z^{*}\right)}=-\frac{1}{\rho} \frac{\partial\left(\rho U^{2} p^{*}\right)}{\partial\left(H x^{*}\right)}+g \sin \theta+\frac{\mu}{\rho}\left(\frac{\partial^{2}\left(U u^{*}\right)}{\partial\left(H x^{*}\right)^{2}}+\frac{\partial^{2}\left(U u^{*}\right)}{\partial\left(H z^{*}\right)^{2}}\right)
$$

and

$$
\frac{\partial\left(U w^{*}\right)}{\partial\left(\frac{H}{U} t^{*}\right)}+\left(U u^{*}\right) \frac{\partial\left(U w^{*}\right)}{\partial\left(H x^{*}\right)}+\left(U w^{*}\right) \frac{\partial\left(U w^{*}\right)}{\partial\left(H z^{*}\right)}=-\frac{1}{\rho} \frac{\partial\left(\rho U^{2} p^{*}\right)}{\partial\left(H z^{*}\right)}-g \cos \theta+\frac{\mu}{\rho}\left(\frac{\partial^{2}\left(U w^{*}\right)}{\partial\left(H x^{*}\right)^{2}}+\frac{\partial^{2}\left(U w^{*}\right)}{\partial\left(H z^{*}\right)^{2}}\right)
$$

which simplify to

$$
\begin{gathered}
\operatorname{Re}\left(\frac{\partial u^{*}}{\partial t^{*}}+u^{*} \frac{\partial u^{*}}{\partial x^{*}}+w^{*} \frac{\partial u^{*}}{\partial z^{*}}\right)=-\operatorname{Re} \frac{\partial p^{*}}{\partial x^{*}}+\frac{\partial^{2} u^{*}}{\partial x^{* 2}}+\frac{\partial^{2} u^{*}}{\partial z^{* 2}}+3 \\
\operatorname{Re}\left(\frac{\partial w^{*}}{\partial t^{*}}+u^{*} \frac{\partial w^{*}}{\partial x^{*}}+w^{*} \frac{\partial w^{*}}{\partial z^{*}}\right)=-\operatorname{Re} \frac{\partial p^{*}}{\partial z^{*}}+\frac{\partial^{2} w^{*}}{\partial x^{* 2}}+\frac{\partial^{2} w^{*}}{\partial z^{* 2}}-3 \cot \theta,
\end{gathered}
$$

where $R e$ is the Reynolds number which is given by $R e=\frac{\rho Q}{\mu}$. Scaling the continuity equation gives

$$
\frac{\partial\left(U u^{*}\right)}{\partial\left(H x^{*}\right)}+\frac{\partial\left(U w^{*}\right)}{\partial\left(H z^{*}\right)}=0
$$

which reduces to

$$
\frac{\partial u^{*}}{\partial x^{*}}+\frac{\partial w^{*}}{\partial z^{*}}=0
$$


Scaling the temperature and concentration equations yields

$$
\begin{gathered}
\frac{D\left(\Delta T T^{*}\right)}{D\left(\frac{U}{H} t^{*}\right)}=\delta\left(\frac{\Delta T}{H^{2}} \nabla_{*}^{2} T^{*}\right) \\
\frac{D\left(\frac{\sigma_{t}}{\sigma_{c}} \Delta T C^{*}\right)}{D\left(\frac{U}{H} t^{*}\right)}=D_{m}\left(\frac{\sigma_{t} \Delta T}{\sigma_{c} H^{2}} \nabla_{*}^{2} C^{*}+\alpha \frac{\Delta T}{H^{2}} \nabla_{*}^{2} T^{*}\right) .
\end{gathered}
$$

These simplify to become

$$
\begin{gathered}
\operatorname{PrRe}\left(\frac{\partial T^{*}}{\partial t^{*}}+u^{*} \frac{\partial T^{*}}{\partial x^{*}}+w^{*} \frac{\partial T^{*}}{\partial z^{*}}\right)=\frac{\partial^{2} T^{*}}{\partial x^{* 2}}+\frac{\partial^{2} T^{*}}{\partial z^{* 2}} \\
\operatorname{ScRe}\left(\frac{\partial C^{*}}{\partial t^{*}}+u^{*} \frac{\partial C^{*}}{\partial x^{*}}+w^{*} \frac{\partial C^{*}}{\partial z^{*}}\right)=\frac{\partial^{2} C^{*}}{\partial x^{* 2}}+\frac{\partial^{2} C^{*}}{\partial z^{* 2}}+S o\left(\frac{\partial^{2} T^{*}}{\partial x^{* 2}}+\frac{\partial^{2} T^{*}}{\partial z^{* 2}}\right)
\end{gathered}
$$

where $\operatorname{Pr}$ is the Prandtl number defined as $\operatorname{Pr}=\frac{\nu}{\delta}$ while $\nu$ is the kinematic viscosity, $S c$ is called the Schmidt number which is given by $S c=\frac{\nu}{D_{m}}$ and $S o=\alpha \frac{\sigma_{c}}{\sigma_{t}}$ is the Soret number. Applying the scaling to the continuity of normal stress condition at the free surface yields

$$
\begin{aligned}
\rho U^{2} p^{*}=\frac{2 \mu}{\left(1+\left(\frac{\partial\left(H h^{*}\right)}{\partial\left(H x^{*}\right)}\right)^{2}\right)} & {\left[\left(\frac{\partial\left(H h^{*}\right)}{\partial\left(H x^{*}\right)}\right)^{2} \frac{\partial\left(U u^{*}\right)}{\partial\left(H x^{*}\right)}-\frac{\partial\left(H h^{*}\right)}{\partial\left(H x^{*}\right)}\left(\frac{\partial\left(U u^{*}\right)}{\partial\left(H z^{*}\right)}+\frac{\partial\left(U w^{*}\right)}{\partial\left(H x^{*}\right)}\right)+\frac{\partial\left(U w^{*}\right)}{\partial\left(H z^{*}\right)}\right] } \\
& -\frac{\left(\sigma_{\infty}-\sigma_{t}\left(\Delta T T^{*}\right)+\sigma_{c}\left(\frac{\sigma_{t}}{\sigma_{c}} \Delta T C^{*}\right)\right) \frac{\partial^{2}\left(H h^{*}\right)}{\partial\left(H x^{*}\right)^{2}}}{\left(1+\left(\frac{\partial\left(H h^{*}\right)}{\partial\left(H x^{*}\right)}\right)^{2}\right)^{\frac{3}{2}}} \text { at } z^{*}=h^{*}\left(x^{*}, t^{*}\right),
\end{aligned}
$$

which becomes

$$
\begin{array}{r}
p=\frac{2}{R e\left[1+\left(\frac{\partial h^{*}}{\partial x^{*}}\right)^{2}\right]}\left[\left(\frac{\partial h^{*}}{\partial x^{*}}\right)^{2} \frac{\partial u^{*}}{\partial x^{*}}-\frac{\partial h^{*}}{\partial x^{*}}\left(\frac{\partial w^{*}}{\partial x^{*}}+\frac{\partial u^{*}}{\partial z^{*}}\right)+\frac{\partial w^{*}}{\partial z^{*}}\right] \\
-\frac{W e-M\left(T^{*}-C^{*}\right)}{\left(1+\left(\frac{\partial h^{*}}{\partial x^{*}}\right)^{2}\right)^{\frac{3}{2}}} \frac{\partial^{2} h^{*}}{\partial x^{* 2}} \text { at } z^{*}=h^{*}\left(x^{*}, t^{*}\right)
\end{array}
$$

where the dimensionless parameters $M$ and the Weber number, $W e$, are defined as

$$
M=\frac{\sigma_{t} \Delta T}{\rho U^{2} H} \quad \text { and } \quad W e=\frac{\sigma_{\infty}}{\rho U^{2} H} .
$$


Scaling the continuity of tangential stress condition at the free surface leads to

$$
\begin{gathered}
{\left[\frac{\partial\left(\sigma_{\infty}-\sigma_{t}\left(\Delta T T^{*}\right)+\sigma_{c}\left(\frac{\sigma_{t}}{\sigma_{c}} \Delta T C^{*}\right)\right)}{\partial\left(H x^{*}\right)}+\frac{\partial\left(H h^{*}\right)}{\partial\left(H x^{*}\right)}\left(\frac{\partial\left(\sigma_{\infty}-\sigma_{t}\left(\Delta T T^{*}\right)+\sigma_{c}\left(\frac{\sigma_{t}}{\sigma_{c}} \Delta T C^{*}\right)\right)}{\partial\left(H z^{*}\right)}\right)\right] \sqrt{1+\left(\frac{\partial\left(H h^{*}\right)}{\partial\left(H x^{*}\right)}\right)^{2}}} \\
=\mu\left[\left(1-\left(\frac{\partial\left(H h^{*}\right)}{\partial\left(H x^{*}\right)}\right)^{2}\right)\left(\frac{\partial\left(U u^{*}\right)}{\partial\left(H z^{*}\right)}+\frac{\partial\left(U w^{*}\right)}{\partial\left(H x^{*}\right)}\right)+2 \frac{\partial\left(H h^{*}\right)}{\partial\left(H x^{*}\right)}\left(\frac{\partial\left(U w^{*}\right)}{\partial\left(H z^{*}\right)}-\frac{\partial\left(U u^{*}\right)}{\partial\left(H x^{*}\right)}\right)\right] \\
\text { at } z^{*}=h^{*}\left(x^{*}, t^{*}\right)
\end{gathered}
$$

which gives

$$
\begin{array}{r}
-M \operatorname{Re} \sqrt{1+\left(\frac{\partial h^{*}}{\partial x^{*}}\right)^{2}}\left[\frac{\partial\left(T^{*}-C^{*}\right)}{\partial x^{*}}+\frac{\partial h^{*}}{\partial x^{*}}\left(\frac{\partial\left(T^{*}-C^{*}\right)}{\partial z^{*}}\right)\right]=\left(1-\left(\frac{\partial h^{*}}{\partial x^{*}}\right)^{2}\right)\left(\frac{\partial w^{*}}{\partial x^{*}}+\frac{\partial u^{*}}{\partial z^{*}}\right) \\
-4\left(\frac{\partial h^{*}}{\partial x^{*}}\right)\left(\frac{\partial u^{*}}{\partial x^{*}}\right) \text { at } z^{*}=h^{*}\left(x^{*}, t^{*}\right) .
\end{array}
$$

The kinematic condition transforms into

$$
U w^{*}=\frac{\partial\left(H h^{*}\right)}{\partial\left(\frac{H}{U} t^{*}\right)}+\frac{\partial\left(H h^{*}\right)}{\partial\left(H x^{*}\right)}\left(U u^{*}\right) \quad \text { at } \quad z^{*}=h^{*}\left(x^{*}, t^{*}\right)
$$

which simplifies to

$$
w^{*}=\frac{\partial h^{*}}{\partial t^{*}}+u^{*} \frac{\partial h^{*}}{\partial x^{*}} \quad \text { at } \quad z^{*}=h^{*}\left(x^{*}, t^{*}\right) .
$$

Applying the scaling to the Newton's law of cooling yields

$$
-\frac{\lambda}{\sqrt{1+\left(\frac{\partial\left(H h^{*}\right)}{\partial\left(H x^{*}\right)}\right)^{2}}}\left[-\frac{\partial\left(\Delta T T^{*}\right)}{\partial\left(H x^{*}\right)} \frac{\partial\left(H h^{*}\right)}{\partial\left(H x^{*}\right)}+\frac{\partial\left(\Delta T T^{*}\right)}{\partial\left(H z^{*}\right)}\right]=\chi\left(\Delta T T^{*}\right) \quad \text { at } \quad z^{*}=h^{*}\left(x^{*}, t^{*}\right),
$$

which reduces to

$$
B T^{*} \sqrt{1+\left(\frac{\partial h^{*}}{\partial x^{*}}\right)^{2}}=\left(\frac{\partial h^{*}}{\partial x^{*}}\right)\left(\frac{\partial T^{*}}{\partial x^{*}}\right)-\frac{\partial T^{*}}{\partial z^{*}} \text { at } z^{*}=h^{*}\left(x^{*}, t^{*}\right)
$$

where $B=\frac{\chi H}{\lambda}$. The zero mass flux condition at the free surface and at the bottom will be scaled as

$$
\begin{aligned}
-\frac{\partial\left(H h^{*}\right)}{\partial\left(H x^{*}\right)} \frac{\partial\left(\frac{\sigma_{t}}{\sigma_{t}} \Delta T C^{*}\right)}{\partial\left(H x^{*}\right)}+ & \frac{\partial\left(\frac{\sigma_{t}}{\sigma_{t}} \Delta T C^{*}\right)}{\partial\left(H z^{*}\right)}+\alpha\left(-\frac{\partial\left(H h^{*}\right)}{\partial\left(H x^{*}\right)} \frac{\partial\left(\Delta T T^{*}\right)}{\partial\left(H x^{*}\right)}+\frac{\partial\left(\Delta T T^{*}\right)}{\partial\left(H z^{*}\right)}\right)=0 \quad \text { at } \quad z^{*}=h^{*}\left(x^{*}, t^{*}\right), \\
& \frac{\partial\left(\frac{\sigma_{t}}{\sigma_{t}} \Delta T C^{*}\right)}{\partial\left(H z^{*}\right)}+\alpha \frac{\partial\left(\Delta T T^{*}\right)}{\partial\left(H z^{*}\right)}=0 \quad \text { at } \quad z^{*}=0 .
\end{aligned}
$$


These simplify to give

$$
\begin{gathered}
-\left(\frac{\partial h^{*}}{\partial x^{*}}\right)\left(\frac{\partial C^{*}}{\partial x^{*}}\right)+\frac{\partial C^{*}}{\partial z^{*}}+S o\left[-\left(\frac{\partial h^{*}}{\partial x^{*}}\right)\left(\frac{\partial T^{*}}{\partial x^{*}}\right)+\frac{\partial T^{*}}{\partial z^{*}}\right]=0 \quad \text { at } z^{*}=h^{*}\left(x^{*}, t^{*}\right), \\
\frac{\partial C^{*}}{\partial z^{*}}+S o \frac{\partial T^{*}}{\partial z^{*}}=0 \quad \text { at } \quad z^{*}=0 .
\end{gathered}
$$

The remaining boundary conditions at $z^{*}=0$ are non-dimensionalized to give

$$
\begin{gathered}
\frac{\partial u^{*}}{\partial z^{*}}=\frac{1}{\beta} u^{*} \quad \text { at } \quad z^{*}=0, \\
w^{*}=0, \quad T^{*}=1 \quad \text { at } \quad z^{*}=0 .
\end{gathered}
$$

where $\beta=\frac{\sqrt{\kappa}}{\varpi H}$ represents the permeability effect of the porous medium.

Equations (2.23)-(2.35) represent the dimensionless form of the governing equations and the boundary conditions. In the next chapter we will employ this model to investigate the instability of the flow. 


\section{Chapter 3}

\section{Linear Stability Analysis}

The problem (2.23) - (2.35) governing a falling binary liquid film along a slippery heated inclined plate admits a simple solution corresponding to a steady flow uniform in the streamwise direction. In this chapter we obtain this equilibrium solution and investigate its stability by means of a linear analysis. For notational convenience we drop the asterisks in equations (2.23) - (2.35).

\subsection{Equilibrium solution}

The equilibrium solution is obtained by solving the problem under the assumption of $x$ and $t$ independence. Equations (2.23) - (2.35) then reduce to

$$
\begin{gathered}
\frac{\partial w}{\partial z}=0, \quad \frac{\partial^{2} u}{\partial z^{2}}=-3, \quad \frac{\partial p}{\partial z}=-3 \frac{\cot \theta}{R e} \\
\frac{\partial^{2} T}{\partial z^{2}}=0 \quad \text { and } \quad \frac{\partial^{2} C}{\partial z^{2}}=0
\end{gathered}
$$

while at $z=h_{s}$ the conditions are given by

$$
\frac{\partial u}{\partial z}=0, \quad p=0, \quad \frac{\partial C}{\partial z}+S o \frac{\partial T}{\partial z}=0 \quad \text { and } \quad \frac{\partial T}{\partial z}=-B T,
$$

where $h_{s}$ is the equilibrium thickness of the liquid film. At $z=0$ the conditions become

$$
\frac{\partial u}{\partial z}=\frac{1}{\beta} u, \quad w=0, \quad T=1 \quad \text { and } \quad \frac{\partial C}{\partial z}+S o \frac{\partial T}{\partial z}=0 .
$$

Also, without loss of generality we can impose the condition $C=0$ at $z=0$. This corresponds to setting the reference concentration $C_{0}$ to be the concentration at the plate induced by the Soret effect. 
As a result, the equilibrium solution can be written as

$$
\begin{gathered}
w=w_{s}(z) \equiv 0 \\
u=u_{s}(z) \equiv-\frac{3}{2} z^{2}+3 h_{s} z+3 \beta h_{s} \\
p=p_{s}(z) \equiv-3 \frac{\cot \theta}{R e}\left(z-h_{s}\right) \\
T=T_{s}(z) \equiv 1-\left(\frac{B}{1+B h_{s}}\right) z
\end{gathered}
$$

and

$$
C=C_{s}(z) \equiv S o\left(\frac{B}{1+B h_{s}}\right) z
$$

We can determine $h_{s}$ by setting the scaled flow rate to unity, i.e.

$$
\int_{0}^{h_{s}} u_{s}(z) d z=1
$$

This yields

$$
h_{s}^{3}+3 \beta h_{s}^{2}-1=0
$$

and solving for $h_{s}$ gives

$$
h_{s}=\frac{1}{2}\left(4-8 \beta^{3}+4 \sqrt{-4 \beta^{3}+1}\right)^{\frac{1}{3}}+\frac{2 \beta^{2}}{\left(4-8 \beta^{3}+4 \sqrt{-4 \beta^{3}+1}\right)^{\frac{1}{3}}}-\beta .
$$

\subsection{Linear stability theory}

Based on the steady-state solution given by equations (3.1) - (3.5), we can express the perturbed equilibrium solution as follows

$$
\begin{gathered}
h=h_{s}+\eta(x, t), \quad u=u_{s}(z)+\tilde{u}(x, z, t), \quad w=\tilde{w}(x, z, t), \\
p=p_{s}(z)+\tilde{p}(x, z, t), \quad T=T_{s}(z)+\tilde{T}(x, z, t) \quad \text { and } \quad C=C_{s}(z)+\tilde{C}(x, z, t),
\end{gathered}
$$

where $\eta, \tilde{u}, \tilde{w}, \tilde{p}, \tilde{T}$ and $\tilde{C}$ are the added infinitesimal perturbation quantities. We employ this perturbed state into the governing equations (2.23) - (2.35) then linearize with respect to the perturbation variables 
to obtain

$$
\begin{gathered}
\frac{\partial \tilde{u}}{\partial x}+\frac{\partial \tilde{w}}{\partial z}=0 \\
\operatorname{Re}\left(\frac{\partial \tilde{u}}{\partial t}+u_{s} \frac{\partial \tilde{u}}{\partial x}+\tilde{w} \frac{d u_{s}}{d z}\right)=-\operatorname{Re} \frac{\partial \tilde{p}}{\partial x}+\frac{\partial^{2} \tilde{u}}{\partial x^{2}}+\frac{\partial^{2} \tilde{u}}{\partial z^{2}} \\
\operatorname{Re}\left(\frac{\partial \tilde{w}}{\partial t}+u_{s} \frac{\partial \tilde{w}}{\partial x}\right)=-\operatorname{Re} \frac{\partial \tilde{p}}{\partial z}+\frac{\partial^{2} \tilde{w}}{\partial x^{2}}+\frac{\partial^{2} \tilde{w}}{\partial z^{2}} \\
\operatorname{Pr} \operatorname{Re}\left(\frac{\partial \tilde{T}}{\partial t}+u_{s} \frac{\partial \tilde{T}}{\partial x}+\tilde{w} \frac{d T_{s}}{d z}\right)=\frac{\partial^{2} \tilde{T}}{\partial x^{2}}+\frac{\partial^{2} \tilde{T}}{\partial z^{2}}
\end{gathered}
$$

and

$$
\operatorname{ScRe}\left(\frac{\partial \tilde{C}}{\partial t}+u_{s} \frac{\partial \tilde{C}}{\partial x}+\tilde{w} \frac{d C_{s}}{d z}\right)=\frac{\partial^{2} \tilde{C}}{\partial x^{2}}+\frac{\partial^{2} \tilde{C}}{\partial z^{2}}+S o\left(\frac{\partial^{2} \tilde{T}}{\partial x^{2}}+\frac{\partial^{2} \tilde{T}}{\partial z^{2}}\right) .
$$

Transferring the boundary conditions at $z=h_{s}+\eta$ to $z=h_{s}$ and linearizing yields the following conditions at $z=h_{s}$

$$
\begin{gathered}
\tilde{p}-3 \frac{\cot \theta}{R e} \eta-\frac{2}{R e} \frac{\partial \tilde{w}}{\partial z}+\left(W e-M\left(T_{s}-C_{s}\right)\right) \frac{\partial^{2} \eta}{\partial x^{2}}=0 \\
-M \operatorname{Re}\left[\frac{\partial \tilde{T}}{\partial x}-\frac{\partial \tilde{C}}{\partial x}-\frac{B(1+S o)}{1+B h_{s}} \frac{\partial \eta}{\partial x}\right]=\frac{\partial \tilde{w}}{\partial x}+\frac{\partial \tilde{u}}{\partial z}-3 \eta \\
\frac{\partial \tilde{C}}{\partial z}+S o \frac{\partial \tilde{T}}{\partial z}=0 \\
\tilde{w}=\frac{\partial \eta}{\partial t}+u_{s} \frac{\partial \eta}{\partial x}
\end{gathered}
$$

and

$$
\frac{\partial \tilde{T}}{\partial z}=\frac{B^{2} \eta}{1+B h_{s}}-B \tilde{T}
$$


While at $z=0$ the conditions are

$$
\begin{gathered}
\frac{\partial \tilde{C}}{\partial z}+S o \frac{\partial \tilde{T}}{\partial z}=0 \\
\frac{\partial \tilde{u}}{\partial z}=\frac{1}{\beta} \tilde{u}
\end{gathered}
$$

and

$$
\tilde{w}=\tilde{T}=0
$$

We next employ normal modes into the linearized perturbation equations which are defined as

$$
(\tilde{u}, \tilde{w}, \tilde{p}, \tilde{T}, \tilde{C}, \eta)=(\hat{u}(z), \hat{w}(z), \hat{p}(z), \hat{T}(z), \hat{C}(z), \hat{\eta}) e^{i k(x-c t)}
$$

where $k$ represents the perturbation wavenumber which is a real positive number, $c$ is a complex number whose real part $\Re(c)$ denotes the phase speed of the perturbation while the product of the imaginary part $\Im(c)$ and $k$ represents the growth rate. Then the linearized perturbation equations (3.8) - (3.12) can be written as

$$
\begin{gathered}
D \hat{w}+i k \hat{u}=0 \\
\operatorname{Re}\left[i k\left(u_{s}-c\right) \hat{u}+D u_{s} \hat{w}\right]=-i k \operatorname{Re} \hat{p}+D^{2} \hat{u}-k^{2} \hat{u} \\
i k \operatorname{Re}\left(u_{s}-c\right) \hat{w}=-\operatorname{Re} \hat{p}+D^{2} \hat{w}-k^{2} \hat{w} \\
\operatorname{Pr} \operatorname{Re}\left[i k\left(u_{s}-c\right) \hat{T}+D T_{s} \hat{w}\right]=D^{2} \hat{T}-k^{2} \hat{T} \\
S c R e\left[i k\left(u_{s}-c\right) \hat{C}+D C_{s} \hat{w}\right]=D^{2} \hat{C}-k^{2} \hat{C}+S o\left(D^{2} \hat{T}-k^{2} \hat{T}\right),
\end{gathered}
$$

where $D$ denotes the differentiation with respect to $z$ operator. Applying the normal modes defined above to the boundary conditions at $z=h_{s}$ gives

$$
\begin{gathered}
\hat{p}-\frac{3}{R e} \cot \theta \hat{\eta}-\frac{2}{R e} D \hat{w}-k^{2}\left[W e-M\left(1-\frac{B h_{s}(1+S o)}{1+B h_{s}}\right)\right] \hat{\eta}=0 \\
-M \operatorname{Reik}\left[\hat{T}-\hat{C}-\frac{B(1+S o)}{1+B h_{s}} \hat{\eta}\right]=-3 \hat{\eta}+D \hat{u}+i k \hat{w}
\end{gathered}
$$




$$
\begin{gathered}
D \hat{C}+S o D \hat{T}=0 \\
\hat{w}=i k\left(u_{s}-c\right) \hat{\eta} \\
D \hat{T}=\frac{B^{2}}{1+B h_{s}} \hat{\eta}-B \hat{T} .
\end{gathered}
$$

While the boundary conditions evaluated at $z=0$ will take the following form

$$
\begin{gathered}
\hat{w}(0)=\hat{T}(0)=0 \\
D \hat{C}+S o D \hat{T}=0
\end{gathered}
$$

and

$$
D \hat{u}=\frac{1}{\beta} \hat{u} .
$$

The pressure $\hat{p}$ can be eliminated from equations (3.22) and (3.23) to give

$$
\begin{array}{r}
\operatorname{Re}\left[i k \hat{u} D u_{s}+i k\left(u_{s}-c\right) D \hat{u}+D u_{s} D \hat{w}-3 \hat{w}\right]+k^{2} \operatorname{Re}\left(u_{s}-c\right) \hat{w} \\
=D^{3} \hat{u}-k^{2} D \hat{u}-i k D^{2} \hat{w}+i k^{3} \hat{w}
\end{array}
$$

Similarly, eliminating $\hat{p}$ from equations (3.22) and (3.26) gives

$$
\begin{aligned}
-k^{2} \hat{u}+D^{2} \hat{u}-3 i k \cot \theta \hat{\eta}-2 i k D \hat{w}-i k^{3} \operatorname{Re} & {\left[W e-M\left(1-\frac{B h_{s}(1+S o)}{1+B h_{s}}\right)\right] \hat{\eta} } \\
& =\operatorname{Re}\left(i k\left(u_{s}-c\right) \hat{u}\right) \text { at } z=h_{s}
\end{aligned}
$$

In order to satisfy the perturbed continuity equation (3.8) we introduce the stream function $\psi$, which is related to the velocity disturbances $\tilde{u}, \tilde{w}$ by

$$
\tilde{u}(x, z, t)=\frac{\partial \psi}{\partial z}, \tilde{w}(x, z, t)=-\frac{\partial \psi}{\partial x} .
$$

In terms of the normal modes, $\psi$ can be written as

$$
\psi=\Psi(z) e^{i k(x-c t)} .
$$

Then $\hat{u}$ and $\hat{w}$ can be expressed as

$$
\hat{u}(z)=D \Psi, \hat{w}(z)=-i k \Psi .
$$


Consequently, we have the following Orr-Sommerfeld type equations

$$
\begin{gathered}
D^{4} \Psi-\left[i k R e\left(u_{s}-c\right)+2 k^{2}\right] D^{2} \Psi+\left[i k^{3} \operatorname{Re}\left(u_{s}-c\right)+k^{4}-3 i k R e\right] \Psi=0, \\
D^{2} \hat{T}-k^{2} \hat{T}-\operatorname{PrRe}\left[i k\left(u_{s}-c\right) \hat{T}+i k\left(\frac{B}{1+B h_{s}}\right) \Psi\right]=0, \\
D^{2} \hat{C}-k^{2} \hat{C}-S c R e\left[i k\left(u_{s}-c\right) \hat{C}-i k \frac{S o B}{1+B h_{s}} \Psi\right]+S o\left(D^{2} \hat{T}-k^{2} \hat{T}\right)=0 .
\end{gathered}
$$

The boundary conditions at $z=h_{s}$ are

$$
\begin{gathered}
D^{3} \Psi-\left[i k R e\left(u_{s}-c\right)+3 k^{2}\right] D \Psi-\left[3 i k \cot \theta+i k^{3} \operatorname{Re}\left(W e-M\left(\frac{1-B h_{s} S o}{1+B h_{s}}\right)\right)\right] \hat{\eta}=0, \\
D^{2} \Psi+k^{2} \Psi+i k M R e(\hat{T}-\hat{C})-\left(3+i k M R e \frac{B(1+S o)}{1+B h_{s}}\right) \hat{\eta}=0, \\
D \hat{C}+S o D \hat{T}=0, \\
\left(u_{s}-c\right) \hat{\eta}+\Psi=0, \\
D \hat{T}+B \hat{T}-\frac{B^{2}}{1+B h_{s}} \hat{\eta}=0 .
\end{gathered}
$$

While the conditions at $z=0$, are

$$
\begin{gathered}
\Psi=\hat{T}=0, \\
D \hat{C}+S o D \hat{T}=0, \\
D^{2} \Psi-\frac{1}{\beta} D \Psi=0 .
\end{gathered}
$$

The problem given by equations (3.36) - (3.46) constitutes an eigenvalue problem with $c$ being the parameter that is to be assigned characteristic values. Solving for $c$ provides the growth rate of the perturbation with wavenumber $k$ for a given set of flow parameters $(\operatorname{Re}, \operatorname{Pr}, B, S c, S o, M, \theta, \beta, W e)$. A positive value of $\Im(c)$ indicates that the perturbation amplitude grows in time, while if $\Im(c)$ is negative 
then the perturbation is damped. A set of flow parameters for which $\Im(c)=0$ is referred to as the state of neutral stability for the perturbation with wavenumber $k$, and corresponds to the threshold between stability and instability for this perturbation. Regarding the stability of the flow, if all the perturbations are damped then the flow is stable, otherwise it is unstable.

\subsection{Asymptotic solution}

The solution of the eigenvalue problem (3.36) - (3.46) can be obtained by carrying out an asymptotic analysis as $k \rightarrow 0$. First, we expand the perturbations $\Psi, \hat{T}, \hat{C}, \hat{\eta}$ and the eigenvalue $c$ in powers of $k$ as follows

$$
\begin{aligned}
\Psi & =\Psi_{0}(z)+i k \Psi_{1}(z)-k^{2} \Psi_{2}(z), \\
\hat{T} & =\hat{T}_{0}(z)+i k \hat{T}_{1}(z)-k^{2} \hat{T}_{2}(z), \\
\hat{C} & =\hat{C}_{0}(z)+i k \hat{C}_{1}(z)-k^{2} \hat{C}_{2}(z), \\
\hat{\eta} & =\hat{\eta}_{0}+i k \hat{\eta}_{1}-k^{2} \hat{\eta}_{2}, \\
c & =c_{0}+i k c_{1}-k^{2} c_{2} .
\end{aligned}
$$

Substituting into the system of equations (3.36) - (3.46), then we have a hierarchy of problems at different orders of $k$. For $\mathcal{O}(1)$, we obtain

$$
D^{4} \Psi_{0}=0, \quad D^{2} \hat{T}_{0}=0, \quad D^{2} \hat{C}_{0}=0
$$

the boundary conditions at $z=h_{s}$ become

$$
\begin{gathered}
D^{3} \Psi_{0}\left(h_{s}\right)=0, \\
D^{2} \Psi_{0}\left(h_{s}\right)-3 \hat{\eta}_{0}=0, \\
D \hat{C}_{0}\left(h_{s}\right)+S o D \hat{T}_{0}\left(h_{s}\right)=0, \\
\left(u_{s}\left(h_{s}\right)-c_{0}\right) \hat{\eta}_{0}-\hat{\Psi}_{0}\left(h_{s}\right)=0, \\
D \hat{T}_{0}\left(h_{s}\right)+B \hat{T}_{0}\left(h_{s}\right)-\frac{B^{2}}{1+B h_{s}} \hat{\eta}_{0}=0,
\end{gathered}
$$

while the boundary conditions at $z=0$ will take the following form

$$
\Psi_{0}(0)=\hat{T}_{0}(0)=0
$$




$$
\begin{aligned}
D \hat{C}_{0}(0)+S o D \hat{T}_{0}(0) & =0, \\
D^{2} \Psi_{0}(0)-\frac{1}{\beta} D \Psi_{0}(0) & =0 .
\end{aligned}
$$

From equation (3.47), $\hat{T}_{0}$ can be expressed as

$$
\hat{T}_{0}=\bar{A}_{1} z+\bar{A}_{2},
$$

where the constants $\bar{A}_{1}, \bar{A}_{2}$ are determined using the boundary conditions (3.52) and (3.53) giving

$$
\hat{T}_{0}=\frac{B^{2} \hat{\eta}_{0}}{\left(1+B h_{s}\right)^{2}} z .
$$

Similarly, $\hat{C}_{0}$ can be written as

$$
\hat{C}_{0}=C_{1} z+C_{2}
$$

The boundary condition (3.50) is used to determine the constant $C_{1}$;

$$
C_{1}=-\frac{S o B^{2} \hat{\eta}_{0}}{\left(1+B h_{s}\right)^{2}}
$$

while $C_{2}$ will be determined from $\mathcal{O}(k)$ problem as explained later.

Integrating the differential equation $D^{4} \Psi_{0}=0$ and using the boundary conditions (3.48), (3.49), (3.53) and (3.55), we obtain

$$
\Psi_{0}=\frac{3}{2} \hat{\eta}_{0} z^{2}+3 \beta \hat{\eta}_{0} z
$$

Furthermore, $c_{0}$ can be determined using the condition (3.51) and we thus have

$$
c_{0}=3 h_{s}\left(2 \beta+h_{s}\right) .
$$

For $\mathcal{O}(k)$ terms, the concentration equation (3.38) is reduced to

$$
D^{2} \hat{C}_{1}(z)-S c R e\left[\left(-\frac{3}{2} z^{2}+3 h_{s} z+3 \beta h_{s}-c_{0}\right) \hat{C}_{0}(z)-\frac{S o B}{1+B h_{s}} \Psi_{0}(z)\right]+S_{o} D^{2} \hat{T}_{1}(z)=0,
$$

integrating this differential equation then substituting for $\hat{C}_{0}(z)$ and $\Psi_{0}(z)$ as given by equations (3.58), 
(3.59) and (3.60), we obtain

$$
\begin{aligned}
& D \hat{C}_{1}(z)=-\frac{1}{8\left(B^{2} h_{s}^{2}+2 B h_{s}\right)}\left(-24 B^{2} C_{2} \operatorname{ReSc} \beta h_{s}^{3}-12 B^{2} C_{2} \operatorname{ReSch} h_{s}^{3} z^{2}+4 B^{2} C_{2} \operatorname{ReSch}_{s}^{2} z^{3}\right. \\
& +24 B^{2} \operatorname{ReScSo} \beta \hat{\eta}_{0} h_{s} z^{2}+12 B^{2} \operatorname{ReScSo} \hat{\eta}_{0} h_{s} z^{3}-3 B^{2} \operatorname{ReScSo\hat {\eta }_{0}} z^{4}+8 B^{2} C_{2} \operatorname{ReSc} c_{0} h_{s}^{2} z \\
& -4 B^{2} \operatorname{ReScSoc} c_{0} \hat{\eta}_{0} z^{2}-48 B C_{2} \operatorname{ReSc} \beta h_{s}^{2} z-24 B C_{2} \operatorname{ReSc} h_{s}^{2} z^{2}+8 B C_{2} \operatorname{ReSc} h_{s} z^{3} \\
& +12 B \operatorname{ReScSo} \beta \hat{\eta}_{0} z^{2}+4 B \operatorname{ReScSo\hat {\eta }_{0}} z^{3}+8 B^{2} \operatorname{So~}_{s}^{2} D \hat{T}_{1}(z)+16 B C_{2} \operatorname{ReSc} c_{0} h_{s} z
\end{aligned}
$$

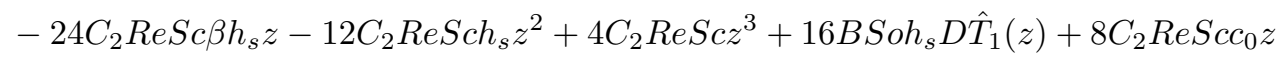

$$
\begin{aligned}
& \left.+8 S o D \hat{T}_{1}(z)\right) \text {. }
\end{aligned}
$$

Form the $\mathcal{O}(k)$ terms, the mass flux conditions (3.41) and (3.45) can be written as

$$
D \hat{C}_{1}\left(h_{s}\right)+S o D \hat{T}_{1}\left(h_{s}\right)=D \hat{C}_{1}(0)+S o \hat{T}_{1}(0)=0,
$$

substituting equation (3.63) into the above conditions, then $C_{2}$ can be determined as

$$
C_{2}=\frac{B S o \eta_{0} h_{s}\left(-24 B \beta h_{s}-9 B h_{s}^{2}+4 B c_{0}-12 \beta-4 h_{s}\right)}{8\left(B h_{s}+1\right)^{2}\left(-3 \beta h_{s}-h_{s}^{2}+c_{0}\right)} .
$$

Consequently, $\hat{C}_{0}$ can be expressed as

$$
\begin{aligned}
\hat{C}_{0}=\frac{1}{8\left(B h_{s}+1\right)^{2}\left(-3 \beta h_{s}-h_{s}^{2}+c_{0}\right)} & \left(\hat { \eta } _ { 0 } S o B \left(-24 B \beta h_{s}^{2}+24 B \beta h_{s} z-9 B h_{s}^{3}+8 B h_{s}^{2} z\right.\right. \\
& \left.\left.+4 B c_{0} h_{s}-8 B c_{0} z-12 \beta h_{s}-4 h_{s}^{2}\right)\right) .
\end{aligned}
$$

At $\mathcal{O}(k)$, we have the following system of equations for $\Psi_{1}$

$$
\begin{gathered}
D^{4} \Psi_{1}(z)-\operatorname{Re}\left(u_{s}(z)-c_{0}\right) D^{2} \Psi_{0}(z)-3 \operatorname{Re} \Psi_{0}(z)=0, \\
D^{3} \Psi_{1}\left(h_{s}\right)-\operatorname{Re}\left(u_{s}\left(h_{s}\right)-c_{0}\right) D \Psi_{0}\left(h_{s}\right)-3 \cot \theta \hat{\eta}_{0}=0, \\
D^{2} \Psi_{1}\left(h_{s}\right)+M \operatorname{Re}\left(\hat{T}_{0}\left(h_{s}\right)-\hat{C}_{0}\left(h_{s}\right)\right)-M \operatorname{Re} \frac{B(S o+1)}{\left(B h_{s}+1\right)} \hat{\eta}_{0}-3 \hat{\eta}_{1}=0, \\
\Psi_{1}\left(h_{s}\right)+\left(u_{s}\left(h_{s}\right)-c_{0}\right) \hat{\eta}_{1}-c_{1} \hat{\eta}_{0}=0, \\
\Psi_{1}(0)=0,
\end{gathered}
$$




$$
D^{2} \Psi_{1}(0)-\frac{1}{\beta} D \Psi_{1}(0)=0 .
$$

Substituting $u_{s}(z), \Psi_{0}(z)$ and $c_{0}$ as given by equations (3.2), (3.60) and (3.61) respectively into the differential equation (3.67) we get

$$
D^{4} \Psi_{1}(z)-9 \operatorname{Re} \hat{\eta}_{0}\left(\beta+h_{s}\right) z+9 \operatorname{Re} \hat{\eta}_{0} h_{s}\left(\beta+h_{s}\right)=0 .
$$

Solving the differential equation leads to

$$
\Psi_{1}(z)=e_{1} z^{3}+e_{2} z^{2}+e_{3} z+e_{4}+\frac{3}{40} \operatorname{Re} \hat{\eta}_{0}\left(\beta+h_{s}\right) z^{5}-\frac{3}{8} \operatorname{Re} \hat{\eta}_{0} h_{s}\left(\beta+h_{s}\right) z^{4}
$$

where $e_{1}, e_{2}, e_{3}$ and $e_{4}$ are integration constants.

Using the condition (3.71) gives $e_{4}=0$ while using the condition (3.72) leads to

$$
e_{3}=2 \beta e_{2}
$$

similarly, using the condition (3.68) and substituting for $u_{s}(z), \Psi_{0}(z)$ and $c_{0}$ we then have

$$
e_{1}=-\frac{3}{2} \operatorname{Re} \beta^{2} \hat{\eta}_{0} h_{s}-\frac{3}{2} \operatorname{Re} \beta \hat{\eta}_{0} h_{s}^{2}+\frac{1}{2} \cot \theta \hat{\eta}_{0} .
$$

The shear stress condition (3.69) is used to determine the constant $e_{2}$ :

$$
\begin{aligned}
e_{2} & =\frac{1}{16\left(3 B^{2} \beta h_{s}^{2}+2 B^{2} h_{s}^{3}+6 B \beta h_{s}+4 B h_{s}^{2}+3 \beta+2 h_{s}\right)}\left(216 B^{2} \operatorname{Re} \beta^{3} \hat{\eta}_{0} h_{s}^{4}+432 B^{2} \operatorname{Re} \beta^{2} \hat{\eta}_{0} h_{s}^{5}\right. \\
& +264 B^{2} \operatorname{Re} \beta \hat{\eta}_{0} h_{s}^{6}+48 B^{2} \operatorname{Re} \hat{\eta}_{0} h_{s}^{7}+432 B \operatorname{Re} \beta^{3} \hat{\eta}_{0} h_{s}^{3}+864 B \operatorname{Re} \beta^{2} \hat{\eta}_{0} h_{s}^{4}+528 B \operatorname{Re} \beta \hat{\eta}_{0} h_{s}^{5} \\
& +96 B \operatorname{Re} \hat{\eta}_{0} h_{s}^{6}+3 b^{2} M \operatorname{ReSo} \hat{\eta}_{0} h_{s}^{2}-72 B^{2} \cot \theta \beta \hat{\eta}_{0} h_{s}^{3}-48 B^{2} \cot \theta \hat{\eta}_{0} h_{s}^{4}+216 \operatorname{Re} \beta^{3} \hat{\eta}_{0} h_{s}^{2} \\
& +432 \operatorname{Re} \beta^{2} \hat{\eta}_{0} h_{s}^{3}+264 \operatorname{Re} \beta \hat{\eta}_{0} h_{s}^{4}+48 \operatorname{Re} \hat{\eta}_{0} h_{s}^{5}+72 B^{2} \beta \hat{\eta}_{1} h_{s}^{2}+48 B^{2} \hat{\eta}_{1} h_{s}^{3}+12 B M \operatorname{ReSo} \beta \hat{\eta}_{0} \\
& +12 B M \operatorname{ReSo} \hat{\eta}_{0} h_{s}-144 B \cot \theta \beta \hat{\eta}_{0} h_{s}^{2}-96 B \cot \theta \hat{\eta}_{0} h_{s}^{3}+24 B M \operatorname{Re} \beta \hat{\eta}_{0}+16 B M \operatorname{Re} \hat{\eta}_{0} h_{s} \\
& \left.+144 B \beta \hat{\eta}_{1} h_{s}+96 B \hat{\eta}_{1} h_{s}^{2}-72 \cot \theta \beta \hat{\eta}_{0} h_{s}-48 \cot \theta \hat{\eta}_{0} h_{s}^{2}+72 \beta \hat{\eta}_{1}+48 \hat{\eta}_{1} h_{s}\right) .
\end{aligned}
$$

As a result, the solution of $\Psi_{1}(z)$ is evaluated by substituting the constants $e_{1}, e_{2}, e_{3}$ and $e_{4}$ into the 
equation (3.74). Furthermore, the kinematic condition (3.70) will be used to determine $c_{1}$ then we get

$$
\begin{aligned}
& c_{1}=\frac{h_{s}}{80}\left(2160 B^{2} R e \beta^{4} h_{s}^{4}+5040 B^{2} R e \beta^{3} h_{s}^{5}+4128 B^{2} \operatorname{Re} \beta^{2} h_{s}^{6}+1440 B^{2} \operatorname{Re} \beta h_{s}^{7}+192 B^{2} R e h_{s}^{8}\right. \\
& +4320 B \operatorname{Re} \beta^{4} h_{s}^{3}+10080 B \operatorname{Re} \beta^{3} h_{s}^{4}+8256 B \operatorname{Re} \beta^{2} h_{s}^{5}+2880 B \operatorname{Re} \beta h_{s}^{6}+384 B \operatorname{Re} h_{s}^{7} \\
& +30 B^{2} M R e S o \beta h_{s}^{2}+15 B^{2} M R e S o h_{s}^{3}-720 B^{2} \cot \theta \beta^{2} h_{s}^{3}-720 B^{2} \cot \theta \beta h_{s}^{4}-160 B^{2} \cot \theta h_{s}^{5} \\
& +2160 \operatorname{Re} \beta^{4} h_{s}^{2}+5040 \operatorname{Re} \beta^{3} h_{s}^{3}+4128 \operatorname{Re} \beta^{2} h_{s}^{4}+1440 \operatorname{Re} \beta h_{s}^{5}+192 \operatorname{Re} h_{6}+120 B M \operatorname{ReSo} \beta^{2} \\
& +180 B M R e S o \beta h_{s}+60 B M R e S o h_{s}^{2}-1440 B \cot \theta \beta^{2} h_{s}^{2}-1440 B \cot \theta \beta h_{s}^{3}-320 B \cot \theta h_{s}^{4} \\
& \left.+240 B M \operatorname{Re} \beta^{2}+280 B M R e \beta h_{s}+80 B M R e h_{s}^{2}-720 \cot \theta \beta^{2} h_{s}-720 \cot \theta \beta h_{2}^{2}-160 \cot \theta h_{s}^{3}\right) / \\
& \left(3 B^{2} \beta h_{s}^{2}+2 B^{2} h_{s}^{3}+6 B \beta h_{s}+4 B h_{s}^{2}+3 \beta+2 h_{s}\right) .
\end{aligned}
$$

The neutral stability state occurs when $\Im(c)=0$ which is equivalent to $c_{1}=0$. Solving for $R e$, then we have the following expression for the critical Reynolds number

$$
\begin{aligned}
R e_{c r i t} & =\left(80\left(3 \beta+h_{s}\right) \cot \theta h_{s}\left(3 B^{2} \beta h_{s}^{2}+2 B^{2} h_{s}^{3}+6 B \beta h_{s}+4 B h_{s}^{2}+3 \beta+2 h_{s}\right)\right) / \\
& \left(2160 B^{2} \beta^{4} h_{s}^{4}+5040 B^{2} \beta^{3} h_{s}^{5}+4128 B^{2} \beta^{2} h_{s}^{6}+1440 B^{2} \beta h_{s}^{7}+192 B^{2} h_{s}^{8}+4320 B \beta^{4} h_{s}^{3}\right. \\
& +10080 B \beta^{3} h_{s}^{4}+8256 B \beta^{2} h_{s}^{5}+2880 B \beta h_{s}^{6}+384 B h_{s}^{7}+30 B^{2} M S o \beta h_{s}^{2}+15 B^{2} M S o h_{s}^{3} \\
& +2160 \beta^{4} h_{s}^{2}+5040 \beta^{3} h_{s}^{3}+4128 \beta^{2} h_{s}^{4}+1440 \beta h_{s}^{5}+192 h_{s}^{6}+120 B M S o \beta^{2}+180 B M S o \beta h_{s} \\
& \left.+60 B M S o h_{s}^{2}+240 B M \beta^{2}+280 B M \beta h_{s}+80 B M h_{s}^{2}\right) .
\end{aligned}
$$

For the impermeable substrate case, $\beta=0$, this expression reduces to

$$
\operatorname{Re}_{\text {crit }}=\frac{10(1+B)^{2} \cot \theta}{12(1+B)^{2}+\frac{5}{12} M B(16+12 S o+3 S o B)},
$$

which is in full agreement with the result obtained by Hu et al. [21] provided the difference in scaling is taken into account. The Soret effect can be discarded by setting $S o=0$, in which case we obtain

$$
\operatorname{Re}_{\text {crit }}=\frac{5}{6} \cot \theta\left[\frac{\left(h_{s}+3 \beta\right) h_{s}}{\frac{1}{2} h_{s}^{2}\left(\beta+h_{s}\right)\left(2 h_{s}^{2}+10 \beta h_{s}+15 \beta^{2}\right)+\frac{5 M B\left(h_{s}+2 \beta\right)}{12\left(1+B h_{s}\right)^{2}}}\right] .
$$

If we use the thickness of equilibrium flow as the length scale, in which case $h_{s}=1$, this expression for the critical Reynolds number will take the following form

$$
\operatorname{Re}_{\text {crit }}=\frac{5}{6} \cot \theta\left[\frac{(1+3 \beta)}{1+6 \beta+\frac{25}{2} \beta^{2}+\frac{15}{2} \beta^{3}+\frac{5 M B(1+2 \beta)}{12(1+B)^{2}}}\right],
$$

which is in full agreement with the result found by Sadiq et al. [16]. If we set $M=S o=\beta=0$, the expression in (3.75) reduces to $R e_{\text {crit }}=\frac{5}{6} \cot \theta$, which is the well known isothermal result obtained by 
Benjamin [3] and Yih [4].

\subsection{Numerical solution}

The eigenvalues of the problem (3.36) - (3.46) will be calculated by employing a Chebyshev spectral collocation method. First, the equations (3.36) - (3.38) will be rewritten as

$$
\begin{gathered}
D^{4} \Psi-\left(i k R e u_{s}+2 k^{2}\right) D^{2} \Psi+\left(i k^{3} \operatorname{Reu}_{s}+k^{4}-3 i k \operatorname{Re}\right) \Psi=c\left(i k^{3} \operatorname{Re} \Psi-i k \operatorname{Re} D^{2} \Psi\right), \\
D^{2} \hat{T}-k^{2} \hat{T}-i k \operatorname{Pr} \operatorname{Re} u_{s} \hat{T}-i k \operatorname{Pr} \operatorname{Re} \frac{B}{1+B h_{s}} \Psi=c(-i k \operatorname{Pr} \operatorname{Re} \hat{T}), \\
D^{2} \hat{C}-k^{2} \hat{C}-i k S c \operatorname{Reu}_{s} \hat{C}+i k S c R e \frac{S o B}{1+B h_{s}} \Psi+S o D^{2} \hat{T}-S o k^{2} \hat{T}=c(-i k S c \operatorname{Re} \hat{C}) .
\end{gathered}
$$

The domain $z \in\left[0, h_{s}\right]$ is shifted to $\xi \in[-1,1]$, by means of the transformation

$$
\xi=\frac{2}{h_{s}} z-1, \frac{d}{d z}=S \frac{d}{d \xi} \quad \text { where } \quad S=\frac{2}{h_{s}} .
$$

We discretize the interval $\xi \in[-1,1]$ using the Chebyshev points

$$
\xi_{l}=-\cos \left(\frac{l \pi}{N}\right), l=0,1,2, \ldots, N
$$

and expand the variables $\Psi(z), \hat{T}(z), \hat{C}(z)$ in terms of the cardinal functions relative to these points as

$$
\Psi=\sum_{j=0}^{N} w_{j} P_{j}(\xi), \hat{T}=\sum_{j=0}^{N} v_{j} P_{j}(\xi), \hat{C}=\sum_{j=0}^{N} u_{j} P_{j}(\xi),
$$

where

$$
P_{j}(\xi)=\frac{\prod_{\substack{n=0 \\ n \neq j}}^{N}\left(\xi-\xi_{n}\right)}{\prod_{\substack{n=0 \\ n \neq j}}^{N}\left(\xi_{j}-\xi_{n}\right)}, j=0,1,2, \cdots, N
$$


Substituting into equation (3.79) and evaluating at the grid points $\xi_{l}, l=0,1,2, \ldots, N$, then we have

$$
\begin{aligned}
S^{4} \sum_{j=0}^{N} w_{j} P_{j}^{(i v)}\left(\xi_{l}\right) & -S^{2} a_{1}\left(\xi_{l}\right) \sum_{j=0}^{N} w_{j} P_{j}^{\prime \prime}\left(\xi_{l}\right)+a_{2}\left(\xi_{l}\right) \sum_{j=0}^{N} w_{j} P_{j}\left(\xi_{l}\right) \\
& =c\left(a_{3} \sum_{j=0}^{N} w_{j} P_{j}\left(\xi_{l}\right)-a_{4} S^{2} \sum_{j=0}^{N} w_{j} P_{j}^{\prime \prime}\left(\xi_{l}\right)\right),
\end{aligned}
$$

where $a_{1}(\xi), a_{2}(\xi), a_{3}$ and $a_{4}$ are defined as

$$
\begin{aligned}
a_{1}(\xi) & =i k \operatorname{Reu}_{s}(\xi)+2 k^{2}, \\
a_{2}(\xi) & =i k^{3} \operatorname{Reu}_{s}(\xi)+k^{4}-3 i k \operatorname{Re}, \\
a_{3} & =i k^{3} \operatorname{Re} \\
a_{4} & =i k R e
\end{aligned}
$$

Now, in terms of $\xi$ the equilibrium velocity is given by

$$
u_{s}(\xi)=\left(-\frac{3}{8} \xi^{2}+\frac{3}{4} \xi+\frac{9}{8}\right) h_{s}^{2}+3 \beta h_{s}
$$

Furthermore, equation (3.82) can be written in matrix form as

$$
S^{4} \bar{D}^{4} \bar{w}-S^{2} A_{1} \bar{D}^{2} \bar{w}+A_{2} \bar{w}=c\left(A_{3}-A_{4}\right) \bar{w}
$$

where $\bar{w}=\left[\begin{array}{lllll}w_{0} & w_{1} & w_{2} & \cdots & w_{N}\end{array}\right]^{T}$ and $\bar{D}$ denotes the differentiation matrix which is defined as

$$
\bar{D}=\left[\begin{array}{ccccc}
P_{0}^{\prime}\left(\xi_{0}\right) & P_{1}^{\prime}\left(\xi_{0}\right) & P_{2}^{\prime}\left(\xi_{0}\right) & \ldots & P_{N}^{\prime}\left(\xi_{0}\right) \\
P_{0}^{\prime}\left(\xi_{1}\right) & P_{1}^{\prime}\left(\xi_{1}\right) & P_{2}^{\prime}\left(\xi_{1}\right) & \ldots & P_{N}^{\prime}\left(\xi_{1}\right) \\
\vdots & \vdots & \vdots & \vdots & \vdots \\
P_{0}^{\prime}\left(\xi_{N}\right) & P_{1}^{\prime}\left(\xi_{N}\right) & P_{2}^{\prime}\left(\xi_{N}\right) & \ldots & P_{N}^{\prime}\left(\xi_{N}\right)
\end{array}\right]
$$

while the matrices $A_{1}, A_{2}, A_{3}$ and $A_{4}$ are given by

$$
\begin{aligned}
& A_{1}=\operatorname{diag}\left(a_{1}\left(\xi_{0}\right), a_{1}\left(\xi_{1}\right), \cdots, a_{1}\left(\xi_{N}\right)\right), \\
& A_{2}=\operatorname{diag}\left(a_{2}\left(\xi_{0}\right), a_{2}\left(\xi_{1}\right), \cdots, a_{2}\left(\xi_{N}\right)\right), \\
& A_{3}=a_{3} I \\
& A_{4}=a_{4} S^{2} \bar{D}^{2} .
\end{aligned}
$$

where $I$ is the $(N+1) \times(N+1)$ identity matrix. 
Similarly, equation (3.80) can be expressed as

$$
-S^{2} \sum_{j=0}^{N} v_{j} P_{j}^{\prime \prime}\left(\xi_{l}\right)+a_{5}\left(\xi_{l}\right) \sum_{j=0}^{N} v_{j} P_{j}\left(\xi_{l}\right)+a_{6} \sum_{j=0}^{N} w_{j} P_{j}\left(\xi_{l}\right)=c a_{7} \sum_{j=0}^{N} v_{j} P_{j}\left(\xi_{l}\right), l=0,1,2, \cdots, N
$$

where

$$
\begin{aligned}
a_{5}(\xi) & =i k \operatorname{Pr} \operatorname{Re} u_{s}(\xi)+k^{2}, \\
a_{6} & =i k \operatorname{Pr} \operatorname{Re} \frac{B}{1+B h_{s}}, \\
a_{7} & =i k \operatorname{Pr} R e
\end{aligned}
$$

Expressing equation (3.85) in matrix form, then we have

$$
-S^{2} \bar{D}^{2} \bar{v}+A_{5} \bar{v}+A_{6} \bar{w}=c A_{7} \bar{v}
$$

where $\bar{v}=\left[\begin{array}{lllll}v_{0} & v_{1} & v_{2} & \cdots & v_{N}\end{array}\right]^{T}$ while the matrices $A_{5}, A_{6}$ and $A_{7}$ are given by

$$
\begin{aligned}
& A_{5}=\operatorname{diag}\left(a_{5}\left(\xi_{0}\right), a_{5}\left(\xi_{1}\right), \cdots, a_{5}\left(\xi_{N}\right)\right), \\
& A_{6}=a_{6} I \\
& A_{7}=a_{7} I .
\end{aligned}
$$

Substituting the expansions now into equation (3.81) we obtain

$$
\begin{array}{r}
S^{2} \sum_{j=0}^{N} u_{j} P_{j}^{\prime \prime}\left(\xi_{l}\right)-a_{8}\left(\xi_{l}\right) \sum_{j=0}^{N} u_{j} P_{j}\left(\xi_{l}\right)+a_{9} \sum_{j=0}^{N} w_{j} P_{j}\left(\xi_{l}\right)+ \\
S o S^{2} \sum_{j=0}^{N} v_{j} P_{j}^{\prime \prime}\left(\xi_{l}\right)-S o k^{2} \sum_{j=0}^{N} v_{j} P_{j}\left(\xi_{l}\right) \\
=c a_{10} \sum_{j=0}^{N} u_{j} P_{j}\left(\xi_{l}\right), l=0,1,2, \cdots, N
\end{array}
$$

where

$$
\begin{aligned}
a_{8}\left(\xi_{i}\right) & =i k S c R e u_{s}(\xi)+k^{2}, \\
a_{9} & =i k S c R e \frac{S o B}{1+B h_{s}}, \\
a_{10} & =-i k S c R e .
\end{aligned}
$$

Equation (3.87) can be written in matrix form as

$$
S^{2} \bar{D}^{2} \bar{u}-A_{8} \bar{u}+A_{9} \bar{w}+S o S^{2} \bar{D}^{2} \bar{v}-S o k^{2} \bar{v}=c A_{10} \bar{u}, \quad \bar{u}=\left[\begin{array}{lllll}
u_{0} & u_{1} & u_{2} & \cdots & u_{N}
\end{array}\right]^{T}
$$


where the matrices $A_{8}, A_{9}$ and $A_{10}$ are defined by

$$
\begin{aligned}
A_{8} & =\operatorname{diag}\left(a_{8}\left(\xi_{0}\right), a_{8}\left(\xi_{1}\right), \cdots, a_{8}\left(\xi_{N}\right)\right) \\
A_{9} & =a_{9} I \\
A_{10} & =a_{10} I
\end{aligned}
$$

We now incorporate the equations (3.83), (3.86) and (3.88) into one system of equations which is of the form $L \overline{\bar{u}}=c \bar{M} \overline{\bar{u}}$ where

$$
\overline{\bar{u}}=\left[\begin{array}{lllllllllllll}
w_{0} & w_{1} & \cdots & w_{N} & v_{0} & v_{1} & \cdots & v_{N} & u_{0} & u_{1} & \cdots & u_{N} & \hat{\eta}
\end{array}\right]^{T}
$$

The vector $\overline{\bar{u}}$ has $3(N+1)+1$ components. The matrix $L$ can be written as

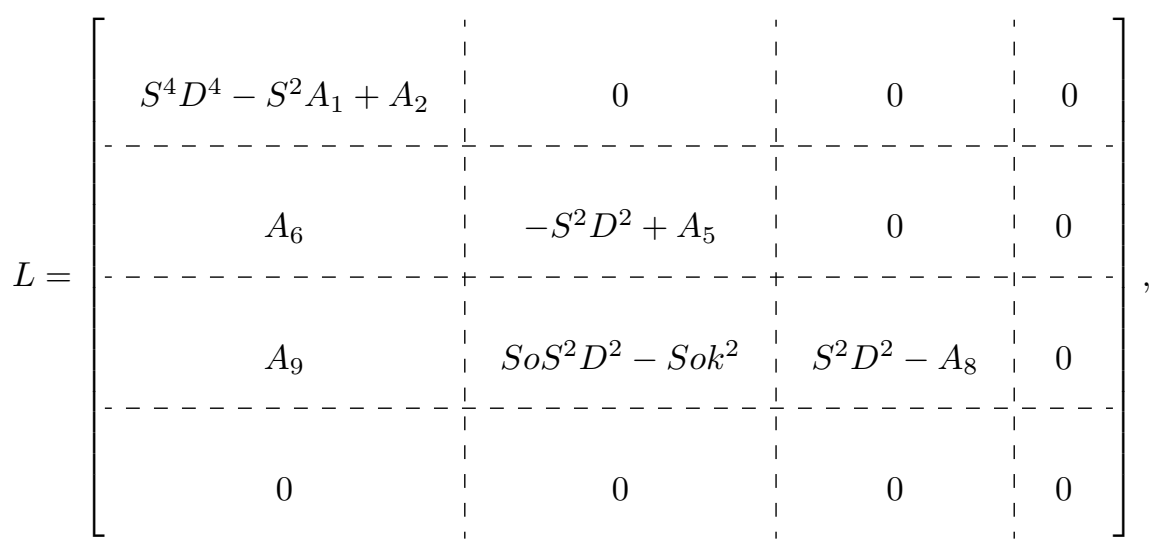

while the matrix $\bar{M}$ is expressed as

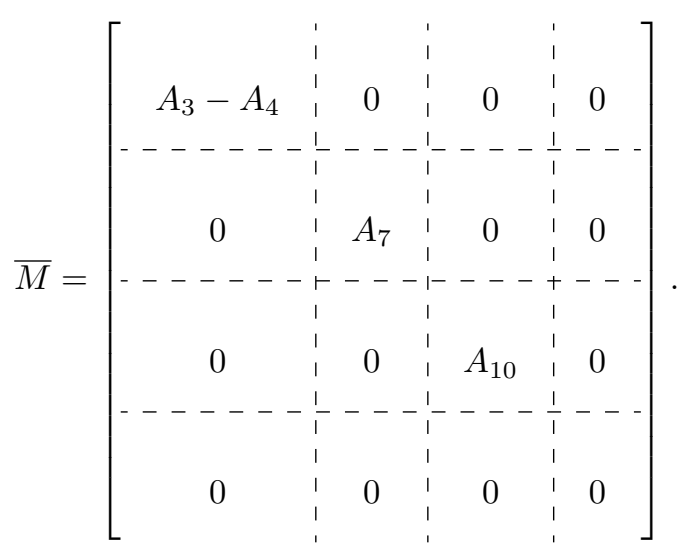

Furthermore, the boundary condition (3.39) can be written in terms of Chebyshev points as follows

$$
S^{3} \sum_{j=0}^{N+1}\left[\bar{D}^{3}\right]_{N+1, j} w_{j}-a_{11} S \sum_{j=0}^{N+1}[\bar{D}]_{N+1, j} w_{j}-a_{12} \hat{\eta}=-c a_{13} S \sum_{j=0}^{N+1}[\bar{D}]_{N+1, j} w_{j}
$$


where $a_{11}, a_{12}$ and $a_{13}$ are defined as

$$
\begin{aligned}
& a_{11}=i k \operatorname{Reu}_{s}\left(\xi_{N+1}\right)+3 k^{2}, \\
& a_{12}=3 i k \cot \theta+i k^{3} \operatorname{Re}\left(W e-M\left(\frac{1-B h_{s} S o}{1+B h_{s}}\right)\right), \\
& a_{13}=i k R e .
\end{aligned}
$$

The boundary conditions given by equations (3.40)-(3.43) are expressed as

$$
\begin{gathered}
S^{2} \sum_{j=0}^{N+1}\left[\bar{D}^{2}\right]_{N+1, j} w_{j}+k^{2} w_{N+1}+a_{14}\left(v_{N+1}-u_{N+1}\right)-a_{15} \hat{\eta}=0, \\
S \sum_{j=0}^{N+1}[\bar{D}]_{N+1, j} u_{j}+S o S \sum_{j=0}^{N+1}[\bar{D}]_{N+1, j} v_{j}=0, \\
w_{N+1}+u_{s}\left(\xi_{N+1}\right) \hat{\eta}=c \hat{\eta}, \\
S \sum_{j=0}^{N+1}[\bar{D}]_{N+1, j} v_{j}+B v_{N+1}-a_{16} \hat{\eta}=0,
\end{gathered}
$$

where $a_{14}, a_{15}$ and $a_{16}$ are given by

$$
\begin{aligned}
& a_{14}=i k M R e, \\
& a_{15}=3+i k M R e\left(\frac{B(1+S o)}{1+B h_{s}}\right), \\
& a_{16}=\frac{B^{2}}{1+B h_{s}} .
\end{aligned}
$$

Similarly, expanding the conditions at $z=0$, we obtain

$$
\begin{gathered}
w_{0}=v_{0}=0 \\
S \sum_{j=0}^{N+1}[\bar{D}]_{1, j} u_{j}+S o S \sum_{j=0}^{N+1}[\bar{D}]_{1, j} v_{j}=0, \\
\beta S^{2} \sum_{j=0}^{N+1}\left[\bar{D}^{2}\right]_{1, j} w_{j}-S \sum_{j=0}^{N+1}[\bar{D}]_{1, j} w_{j}=0 .
\end{gathered}
$$

The transformed boundary conditions (3.92)-(3.99) can be incorporated into the system $L \overline{\bar{u}}=c \bar{M} \overline{\bar{u}}$ by replacing certain rows in the system.

It turns out that the parameters $W e, M$ and $B$ are implicity dependent on the Reynolds number. Thus, in order to determine the solution for the critical Reynolds number we introduce new parameters 
$K a, M a$ and $B i$ related to the previous ones by

$$
\begin{gathered}
W e=\left(\frac{3}{\sin \theta}\right)^{1 / 3} \frac{K a}{R e^{5 / 3}}, \\
M=\left(\frac{3}{\sin \theta}\right)^{1 / 3} \frac{M a}{R e^{5 / 3}}, \\
B=\left(\frac{3}{\sin \theta}\right)^{1 / 3} B i R e^{1 / 3},
\end{gathered}
$$

where $K a, M a$ and $B i$ are defined as

$$
\begin{aligned}
K a & =\frac{\sigma_{\infty} \rho^{1 / 3}}{g^{1 / 3} \mu^{4 / 3}} \quad \text { is the Kapitza number, } \\
M a & =\frac{\sigma_{t} \Delta T \rho^{1 / 3}}{g^{1 / 3} \mu^{4 / 3}} \quad \text { is the Marangoni number, and } \\
B i & =\frac{\chi \mu^{2 / 3}}{\lambda g^{1 / 3} \rho^{2 / 3}} \quad \text { is the Biot number. }
\end{aligned}
$$

In terms of the new parameters, the asymptotic expression for neutral stability yields two solutions for the critical Reynolds number. These correspond to the so-called S and H modes of instability. For small Reynolds numbers, inertial forces are insufficient to amplify waves on the surface of the fluid layer. However, the equilibrium flow may still be unstable due to flow induced by Marangoni stresses resulting from surface tension variation caused by perturbations in temperature and solute concentration. The amplification of perturbations much longer than the thickness of the layer by this type of instability is referred to as the $\mathrm{S}$ mode.

For sufficiently strong inertia perturbations in the elevation of the surface of the liquid are amplified leading to a hydrodynamic instability referred to as the $\mathrm{H}$ mode. Thermocapillary and solutocapillary effects can enhance the instability of the $\mathrm{H}$ mode if the variation in surface tension results in stronger surface tension at the crests of surface waves than at the troughs. Marangoni stresses then pull fluid towards the crests thus amplifying the amplitude.

Neutral stability curves in the $k-R e$ plane are shown in Figures $3.1-3.4$. The intercepts with the $R e$-axis correspond to the critical Reynolds number for the onset of instability of perturbations with $k=0$. These are in excellent agreement with the result from the asymptotic analysis when the parameters are $\mathcal{O}(1)$. The results reveal that as $M a$ is increased, both the $\mathrm{S}$ and $\mathrm{H}$ modes are destabilized and the two modes eventually merge indicating that the equilibrium flow is unstable for all Reynolds numbers. The parameter $M a$ is a measure of thermocapillarity, so as it is increased temperature perturbations cause greater surface tension variation which destabilizes the flow. Furthermore, if the free surface is undulated, then the troughs are warmer than the crests due to their proximity to the heated bottom. As a result surface tension is stronger at the crests and thus the resulting Marangoni stresses act to amplify the undulations and as such the $\mathrm{H}$ mode is destabilized. 


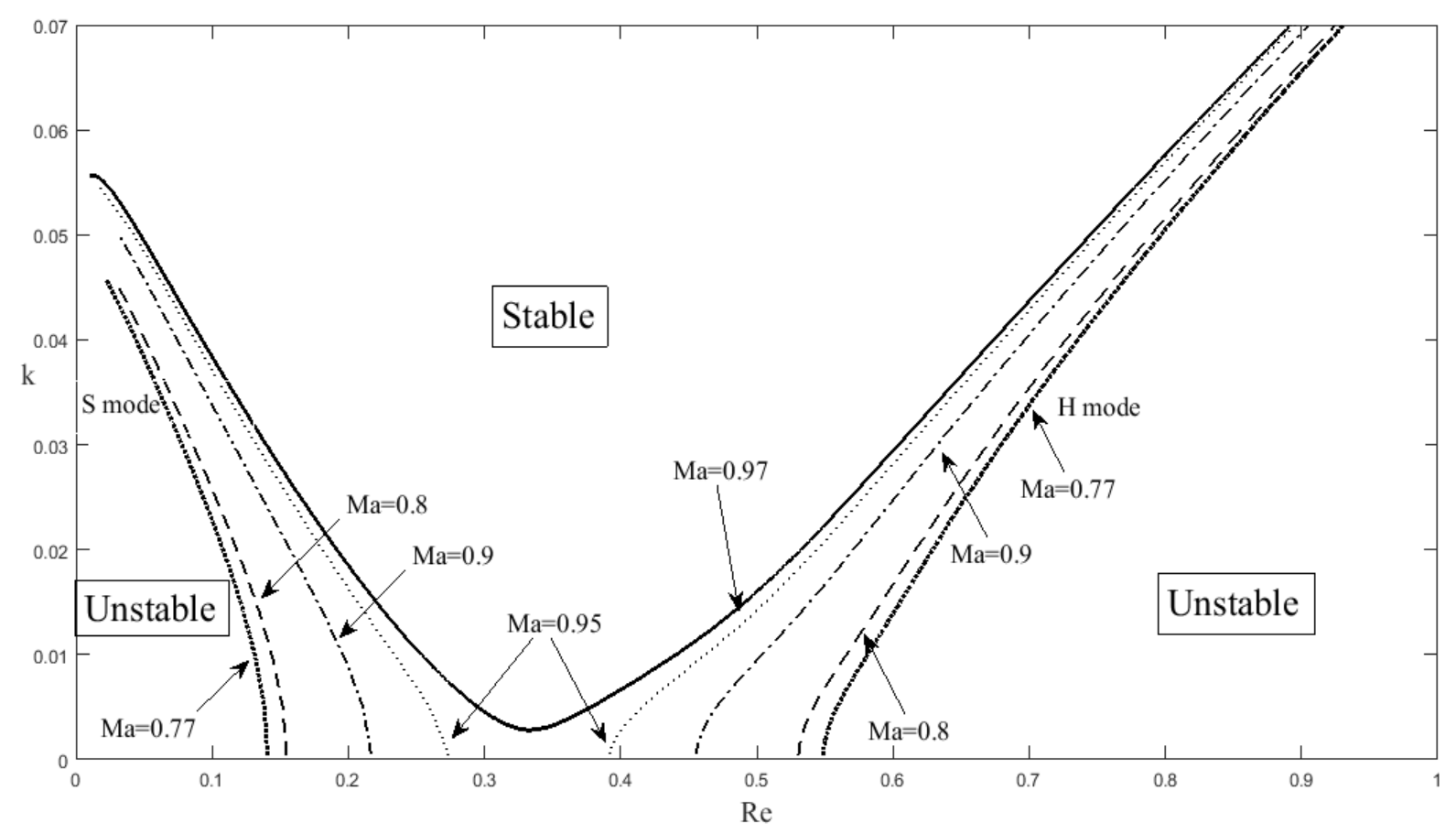

Figure 3.1: Neutral stability curves for different values of $M a$ with $\cot \theta=1, \operatorname{Pr}=7, B i=1, K a=$ $100, S c=700, S o=0.5$ and $\beta=0$.

The results in Figure 3.1 and 3.2 correspond to the impermeable substrate case, $\beta=0$. Note that in Figure 3.1 the onset of instability of the equilibrium flow is due to the amplification of infinitely long perturbations. However, it turns out that if $S o$ is negative and of sufficiently large absolute value, the onset of instability is due to the amplification of a perturbation of finite wavelength as shown in Figure 3.2. The results in Figures 3.3 and 3.4 correspond to a case with a permeable bottom, respectively for a positive and negative value for So. As it can be seen, like in the impermeable case, with negative So instability of the equilibrium flow is due to finite wavelength perturbations. We conclude that this phenomenon is not qualitatively affected by substrate permeability.

Another observation from the neutral stability curves is that for a given Marangoni number the interval of Reynolds numbers for which the flow is stable is smaller if the substrate is permeable suggesting that substrate permeability is a destabilizing factor. To better determine how permeability affects the stability of the flow, in Figure 3.5 we display the critical Reynolds number as a function of $M a$ for different values of the permeability of the substrate parameter $\beta$. The upper branch of the curve describes the onset of the instability of the $\mathrm{H}$ mode while the $\mathrm{S}$ mode is described by the lower branch of the curve, and the region inside the curve indicates the Reynolds numbers for which the flow is stable. It can be seen that the region of stability shrinks as $\beta$ is increased, as a result we conclude that increasing the 


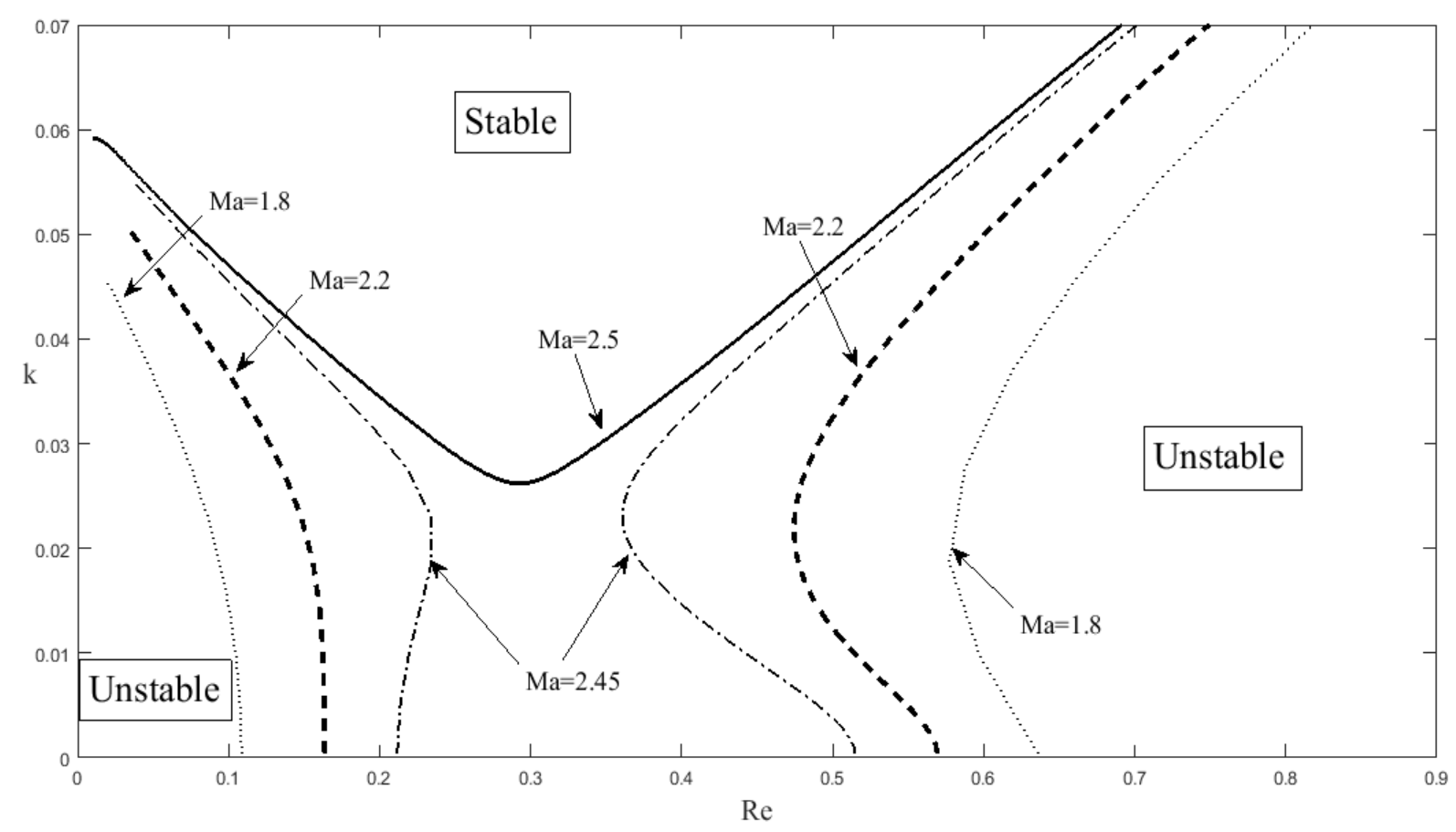

Figure 3.2: Neutral stability curves for different values of $M a$ with $\cot \theta=1, \operatorname{Pr}=7, B i=1, K a=$ $100, S c=700, S o=-0.5$ and $\beta=0$.

permeability of the substrate destabilizes the flow for both the $\mathrm{S}$ and $\mathrm{H}$ modes. These conclusions are the same as those observed by Sadiq et al. [16] for the thermo-porous problem without the Soret effect. 


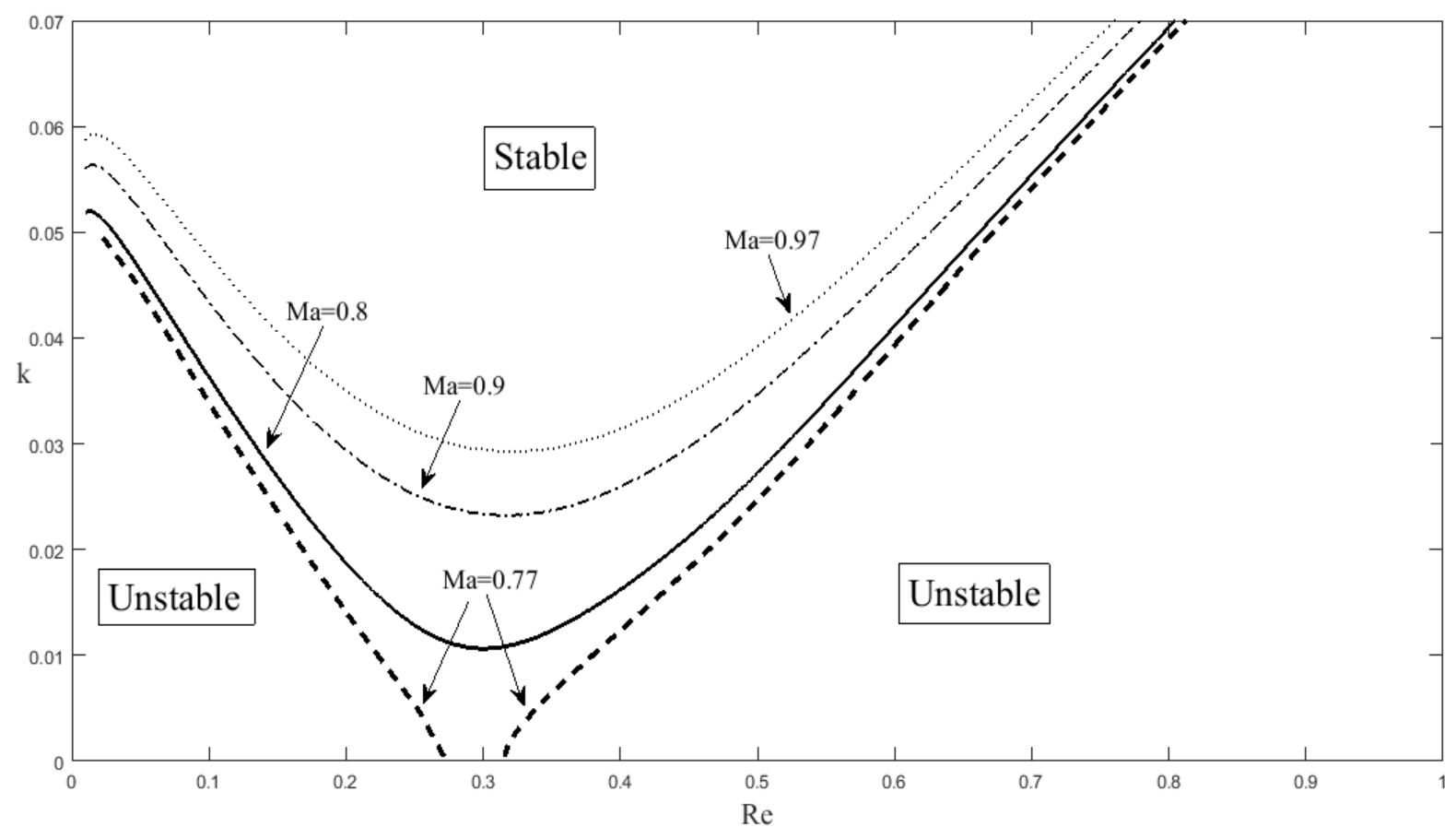

Figure 3.3: Neutral stability curves for different values of $M a$ with $\cot \theta=1, \operatorname{Pr}=7, B i=1, K a=$ $100, S c=700, S o=0.5$ and $\beta=0.2$. 


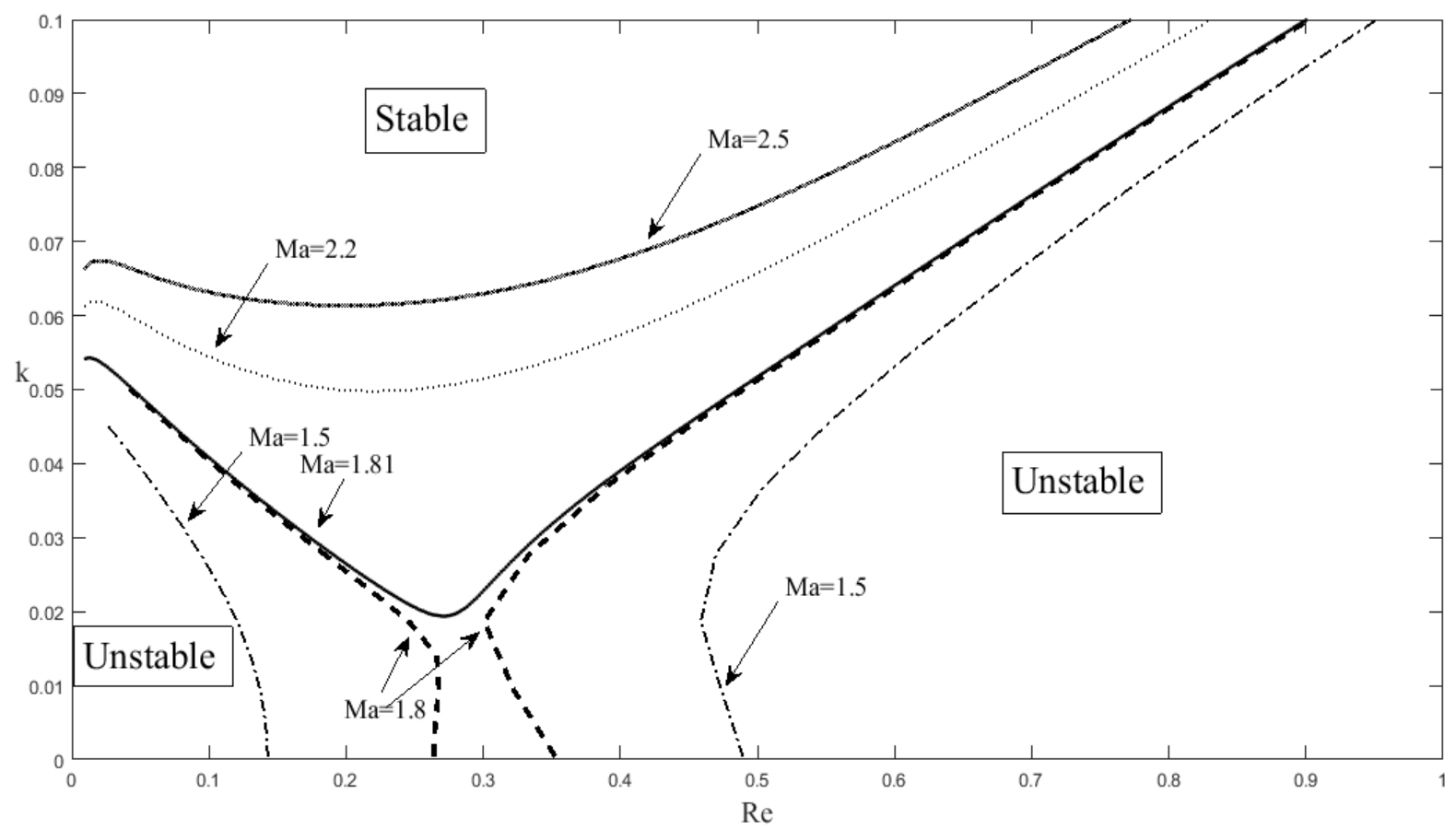

Figure 3.4: Neutral stability curves for different values of $M a$ with $\cot \theta=1, \operatorname{Pr}=7, B i=1, K a=$ $100, S c=700, S o=-0.5$ and $\beta=0.2$. 


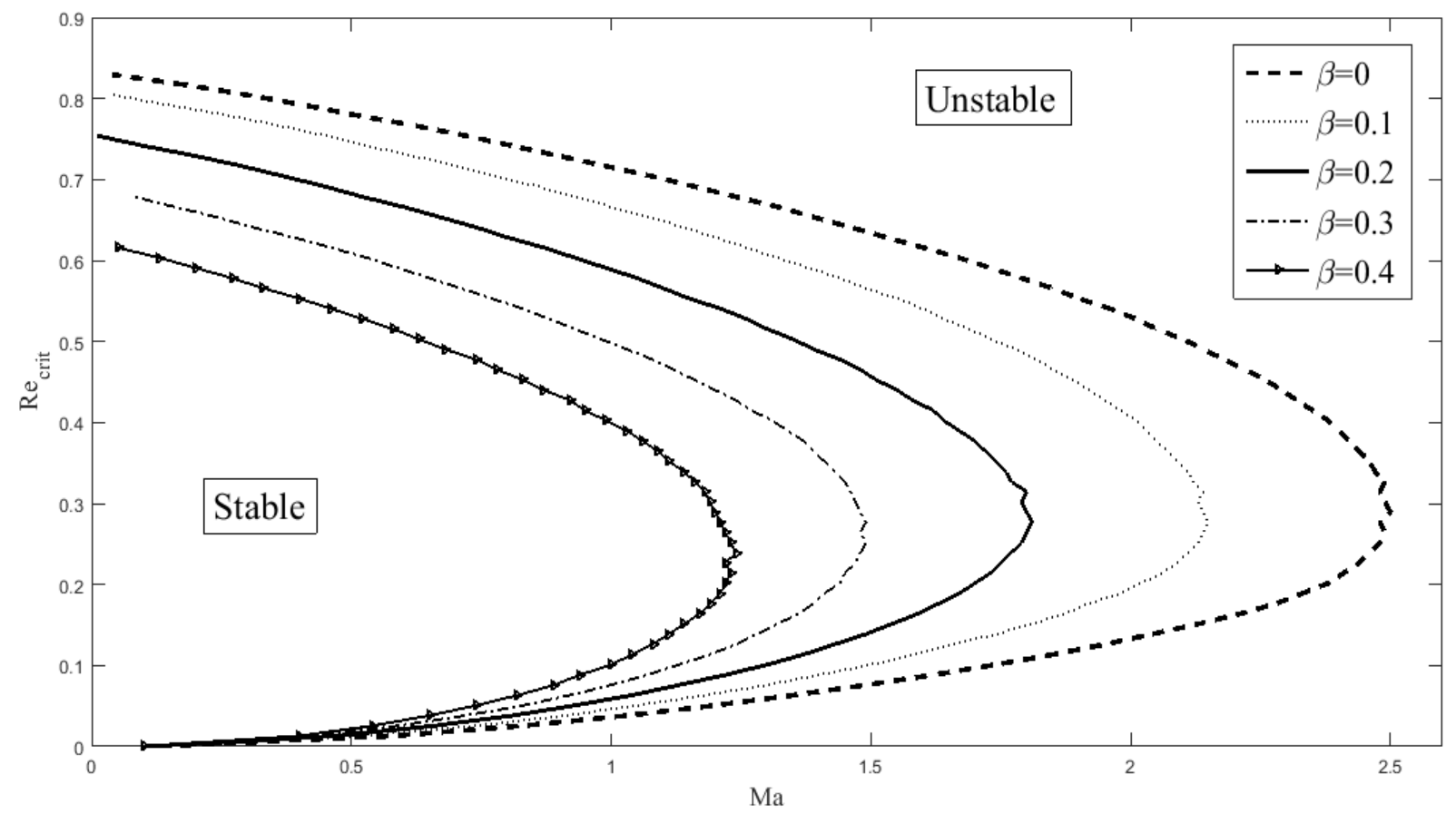

Figure 3.5: $R e_{\text {crit }}$ as function of $M a$ with $\cot \theta=1, \operatorname{Pr}=7, B i=1, K a=100, S c=700$ and $S o=$ -0.5 . 


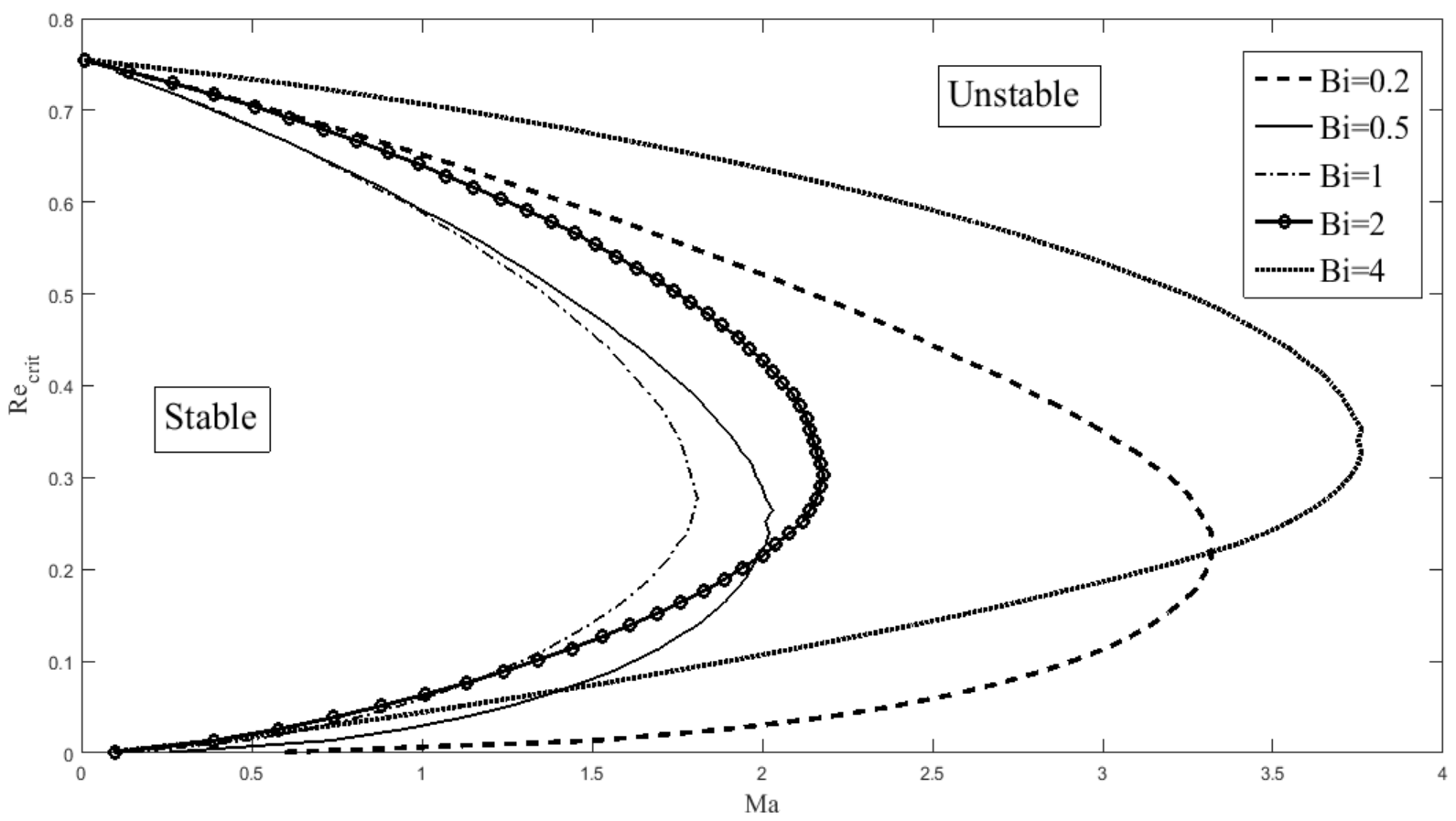

Figure 3.6: $R e_{\text {crit }}$ as function of $M a$ with $\cot \theta=1, \operatorname{Pr}=7, K a=100, S c=700, S o=-0.5$ and $\beta=$ 0.2 .

The effect of the Biot number on the critical Reynolds number is illustrated in Figure 3.6. In fact when $B i=0$ the equilibrium temperature is constant for the fluid layer including the surface. As a result the surface temperature and the concentration of solute are uniform. Consequently, the Marangoni effect and the Soret effect are neutralized.

On the other hand, for large Biot numbers the surface temperature approaches that of the ambient gas which is constant and as such the Marangoni effect and the Soret effect are again neutralized. Therefore, there is a critical Biot number for which surface tension varies the most because of largest temperature and concentration variations. At this value the Marangoni effect is maximized resulting in the most unstable flow. As observed from the curves in Figure 3.6, when $B i$ increases from zero the region of stability shrinks, reaches a minimum and then increases. As a result we conclude that both $\mathrm{S}$ and $\mathrm{H}$ mode experience a destabilizing effect with small values of $B i$ and a stabilizing effect with sufficiently large values of $B i$. 


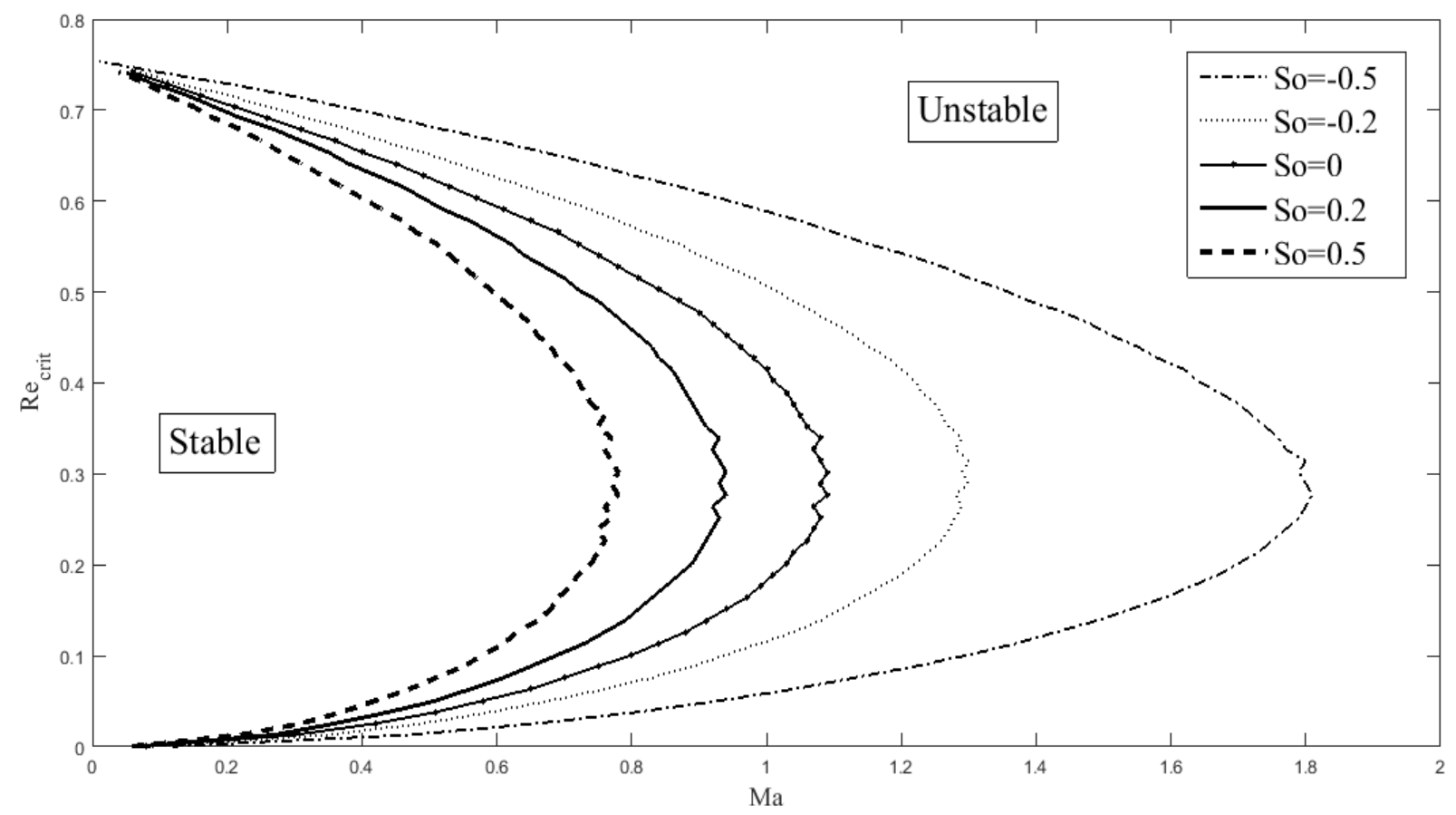

Figure 3.7: Re $e_{c r i t}$ as function of $M a$ with $\cot \theta=1, \operatorname{Pr}=7, B i=1, K a=100, S c=700$ and $\beta=0.2$.

Figure 3.7 shows $R e_{\text {crit }}$ versus $M a$ for different values of the Soret number holding the other flow parameters constant. The results illustrate that increasing the Soret number destabilizes the flow. More specifically, if $S o$ is positive increasing the magnitude of the Soret effect, measured by the absolute value of $S o$, destabilizes the flow, and if $S o$ is negative increasing the Soret effect stabilizes the flow. In fact, increasing the magnitude of the Soret effect results in greater concentration differences induced by temperature perturbations and thus amplifies the Marangoni stresses. However, if $S o$ is negative a mass flux is induced towards warmer regions and as a result we have a higher concentration of solute at the troughs of surface perturbations than at the crests. But surface tension increases with concentration so, with negative values for $S o$, the Soret effect acts to increase surface tension at the troughs and lower it at the crests which acts to dampen surface undulations and stabilize the flow.

We next consider the effect of the magnitude of the surface tension which is measured by the Kapitza number. Now, it is well known that surface tension dampens surface waves, but it has very little effect on very long waves. For cases when $S o$ is positive we do not expect any effect of the Kapitza number on the onset of instability since it is due to the amplification of infinitely long perturbations. For cases when $S o$ is negative, like the one considered in Figure 3.8, the onset of instability is due to perturbations of finite wavelength. So the region of stability increases with $K a$. 


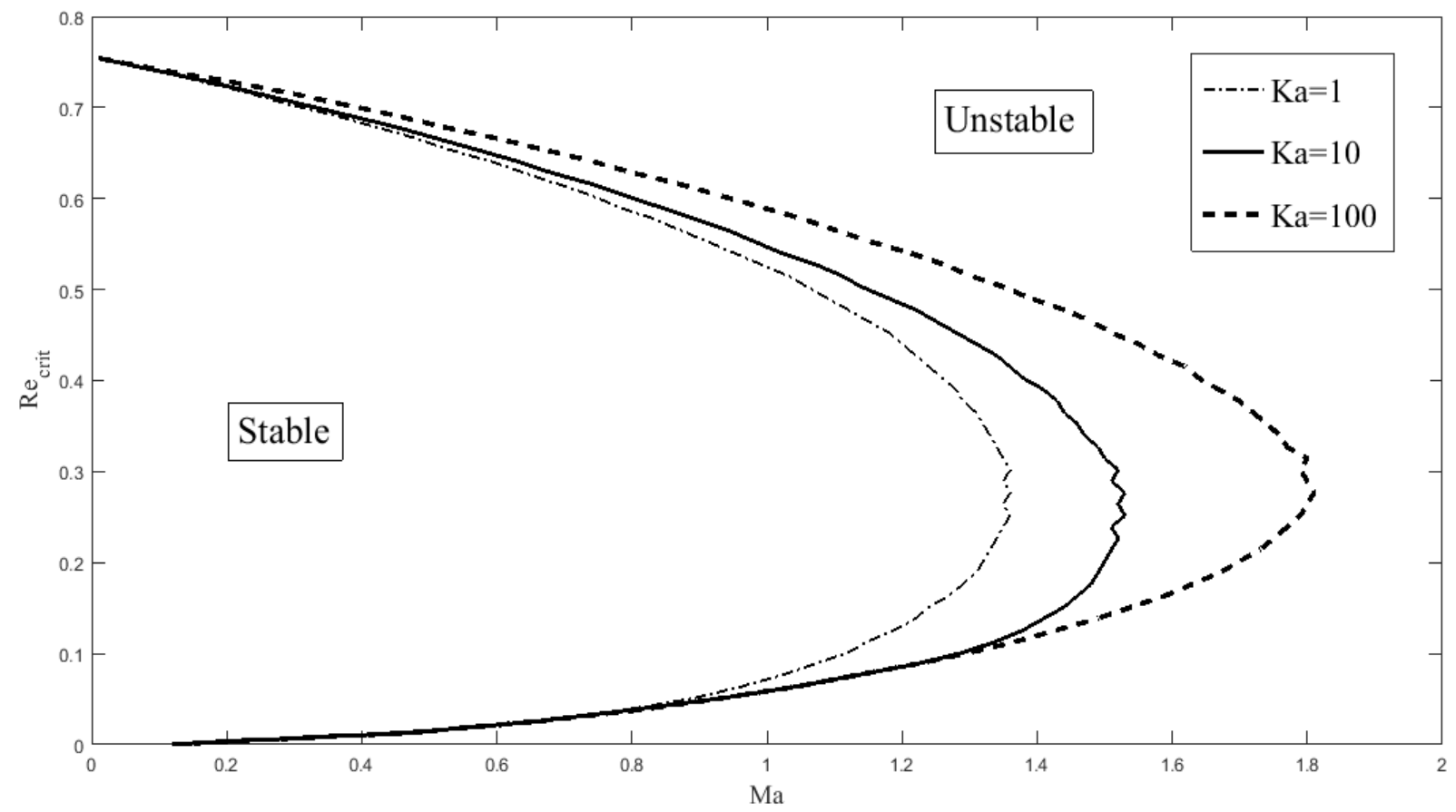

Figure 3.8: Re $e_{c r i t}$ as function of $M a$ with $\cot \theta=1, \operatorname{Pr}=7, B i=1, S c=700, S o=-0.5$ and $\beta=0.2$. 


\section{Chapter 4}

\section{Conclusions}

In this thesis we studied the instability of the flow of a binary liquid film down a uniformly heated porous inclined plane. A mathematical model was constructed by coupling the Navier-Stokes equations governing the flow with concentration and temperature equations. The permeability of the substrate was incorporated by applying a slip condition at the bottom of the liquid layer. Our goal was to determine the effect of various flow parameters such as the Soret number, permeability of the substrate, Biot number and Kapitza number on the critical Reynolds number for instability.

The solution for the equilibrium state was obtained, then we introduced a perturbed state imposed on the equilibrium solution into the governing equations. We linearized the perturbation equations and obtained an eigenvalue problem with an Orr-Sommerfeld type equation. A Chebyshev spectral collocation method was carried out to determine the critical Reynolds number and reveal more information about the onset of instability associated with the various flow parameters. An asymptotic solution was also performed resulting in an excellent agreement with the numerical solution.

Neutral stability curves for flow over permeable and impermeable substrates were obtained for various values of the Marangoni number, Ma. Our investigation showed that the Marangoni number, which is a measure of thermocapillarity, plays a destabilizing role on the flow. More specifically increasing $M a$ destabilizes both the $\mathrm{S}$ and $\mathrm{H}$ modes, and for sufficiently large values of $M a$ the two modes merge rendering the flow unstable for all Reynolds number. Moreover, the results revealed that the onset of instability is because of the amplification of infinitely-long perturbation when the Soret number is positive. However, when the Soret number is negative the onset of instability is due to the amplification of a perturbation of finite wavelength. It turns out that permeability of the substrate does not change this phenomena.

Our results also indicate that the Soret effect acts as a destabilizing factor when the Soret number is positive, and a stabilizing factor when it is negative. While the permeability of the substrate was found

to play a destabilizing role in all cases, as revealed by the fact that the interval of Reynolds numbers for which the equilibrium flow is stable shrinks as $\beta$ is increased.

An important aspect that we found is how the instability of the flow is affected by increasing the Biot number. The results we obtained show that increasing the Biot number has different effects according 
to its value. When $B i=0$ or when $B i$ is very large both the Marangoni effect and the Soret effect are neutralized since the surface temperature and concentration of the fluid are uniform and as a result the flow is more stable. As such the critical Reynolds number reaches a minimum at an intermediate Biot number. As a result increasing the Biot number from zero to this value destabilizes the flow while a stabilizing effect occurs with larger values of $B i$.

We also found that when the onset of instability is due to infinitely long perturbations, increasing the Kapitza number has no effect. This is due to the fact that the Kapitza number measures the magnitude of surface tension which does not impact long waves due to their reduced curvature. On the other hand, when the Soret number is negative, and the onset of instability is due to the amplification of waves with finite wavelength, the effect of the Kapitza number is significant with increased values resulting in a more stable flow. 


\section{References}

1. Kapitza, P. L. 1948 Wave flow of thin layers of a viscous fluid: II. Fluid flow in the presence of continuous gas flow and heat transfer. Zh. Eksp. Teor. Fiz. 18, 19-28.

2. Kapitza, P. L. and Kapitza, S. P. 1949 Wave flow of thin layers of a viscous fluid: III. Experimental study of undulatory flow conditions. Zh. Eksp. Teor. Fiz. 19, 105-120.

3. Benjamin, T. B. 1957 Wave formation in laminar flow down an inclined plane. J. Fluid Mech. 2, 554-574.

4. Yih, C.-S. 1963 Stability of liquid flow down an inclined plane. Phys. Fluids 6, 321-334.

5. Kalliadasis, S., Ruyer-Quil, C., Scheid, B. and Velarde, M. 2012 Falling liquid films, Springer, London.

6. Ruyer-Quil, C., Scheid, B., Kalliadasis, S., Velarde, M., and Zeytounian, R. 2005 Thermocapillary long waves in a liquid film flow. part 1. low-dimensional formulation. J. Fluid Mech. 538, 199-222.

7. Scheid, B., Ruyer-Quil, C., Kalliadasis, S., Velarde, M., and Zeytounian, R. 2005 Thermocapillary long waves in a liquid film flow. part 2. linear stability and nonlinear waves. J. Fluid Mech. 538, 223-244.

8. Trevelyan, P., Scheid, B.,Ruyer-Quil, C. and Kalliadasis, S. 2007 Heated falling films. J. Fluid Mech. 592, 295-334.

9. D'Alessio, S. J. D., Pascal, J. P., Jasmine, H. A. and Ogden K.A. 2010 Film flow over heated wavy inclined surfaces. J. Fluid Mech. 665, 418-456.

10. Pascal, J. P. 1999 Linear stability of fluid flow down a porous inclined plane. J. Phys. D 32, 417-422.

11. Beavers, G. S. and Joseph, D. D. 1967 Boundary conditions at a naturally permeable wall. J. Fluid Mech. 30, 197-207.

12. Sadiq, I. M. R. and Usha, R. 2008 Thin Newtonian film flow down a porous inclined plane: Stability analysis. Phys. Fluids 20, 022105. 
13. Liu, R. and Liu, Q. S. 2009 Instabilities of a liquid film flowing down an inclined porous plane. Phys. Rev. E 80, 036316.

14. Pascal, J. P. 2006 Instability of power-law fluid flow down a porous incline. J. Non-Newtonian Fluid Mech. 133, 109-120.

15. Pascal, J. P., D'Alessio, S.J.D. 2010 Instabilities in gravity-driven flow over uneven permeable surfaces Int. J. Multiphase Flow 23, 449-459.

16. Sadiq, I. M. R., Usha, R. and Joo, S. 2010 Instabilities in a liquid film flow over an inclined heated porous substrate. Chem. Eng. Sci. 65, 4443.

17. Takashima, M. 1979 Surface tension driven instability in a horizontal layer of binary liquid mixture in the presence of the Soret effect. J. Phys. Soc. Japan 47, 1321-1326.

18. Pearson, J. R. A. 1958 On convection cells induced by surface tension. J. Fluid Mech. 4, 489-500.

19. Joo, S. W. 1995 Marangoni instabilities in liquid mixtures with Soret effects. J. Fluid Mech. 293, 127-145.

20. Podolny, A., Oron, A. and Nepomnyashchy, A. A. 2006 Linear and nonlinear theory of long-wave Marangoni instability with the Soret effect at finite Biot numbers. Phys. Fluids 18, 054104.

21. Hu, J., Ben Hadid, H., Henry, D. and Mojtabi, A. 2008 Linear temporal and spatio-temporal stability analysis of a binary liquid film flowing down an inclined uniformly heated plate. J. Fluid Mech. 599, 269-298. 